Les effets de l'immersion en eau froide ou contrastée sur la récupération post-effort dans les sports acycliques : une revue systématique et une méta-analyse

\author{
PIERRE CARBONE \\ Etudiant HES - Filière Physiothérapie \\ GILLES HUMBERT-DROZ \\ Etudiant HES - Filière Physiothérapie
}

Directeur de travail : NICOLAS MATHIEU

TRAVAIL DE BACHELOR POUR L'OBTENTION DU DIPLÔME

BACHELOR OF SCIENCE HES-SO IN PHYSIOTHERAPIE

JUILLET 2013 


\section{Résumé}

Introduction : Les différentes techniques d'immersion, que ce soit en eau froide (Cold Water Immersion, CWI) ou en eau contrastée (Contrast Water Therapy, CWT) représentent un fort intérêt dans le monde du sport afin d'optimiser la récupération des athlètes. Cependant, un manque de consensus existe quant aux effets et à l'utilité de ces modalités de récupération. Le but de notre travail est d'évaluer les effets de la $C W I$ et de la $C W T$ sur la récupération suite à un effort musculaire dans les sports acycliques.

Méthode : Nous avons exploré la littérature scientifique et réalisé une revue systématique et une méta-analyse. 441 articles ont été répertoriés après consultation de quatre bases de données. L'analyse des articles et l'évaluation de leur qualité nous ont permis d'éliminer 437 études. Au final, quatre études randomisées contrôlées ont été analysées.

Résultats : L'utilisation de la CWI démontre une capacité statistiquement significative de réduction des courbatures (Delayed Onset Muscle Soreness, DOMS). Le taux de créatine-kinase est cliniquement influencé par la CWI. La capacité fonctionnelle des membres inférieurs ne montre pas de différence statistiquement significative en faveur de la $C W I$ ou de la $C W T$. Ces résultats sont valables à 24 heures post-effort.

Conclusion: De manière générale, des améliorations ont été observées suite à l'utilisation de la $C W T$ ou de la $C W I$. Cette dernière démontre notamment une efficacité pour réduire les DOMS à $24 \mathrm{H}$. Cependant, l'échantillon analysé est faible et certains biais existent. De futures recherches sont nécessaires pour évaluer l'immersion comme technique de récupération.

Mots-clés : immersion en eau froide, immersion en eau contrastée, récupération, sport, courbatures, créatine-kinase, capacité fonctionnelle 


\section{Zusammenfassung}

Einführung: Die verschiedenen Techniken des Eintauchens, entweder in kaltes Wasser (Cold Water Immersion, CWI) oder Kalt/Warm-Behandlung (Contrast Water Therapy, CWT) stellen eine interessante Therapie dar, um die Regenerierung der Muskeln nach dem Training des Athleten zu maximieren. Auswirkungen und Nutzen dieser Methoden für eine Erholung werden jedoch kontrovers diskutiert. Das Ziel unserer Studie war es, $\mathrm{zu}$ überprüfen, welche Auswirkungen von der $C W I$ und von der $C W T$ auf die Regenerierung nach Muskelarbeit in azyklischen Sportarten haben.

Methode: Wir führten eine Literaturrecherche und anschließend eine systematische Übersichtsarbeit und Meta-Analyse durch. 441 Artikel wurden durch den Einsatz von vier Datenbanken gefunden. Die Analyse dieser Artikel und die Beurteilung ihrer Qualität haben es uns ermöglicht, vier randomisierte kontrollierte Studien genauer zu analysieren.

Ergebnisse: Die Kaltwasseranwendung CWI reduziert statistisch signifikant verzögert auftretende Muskelschmerzen (Delayed Onset Muscle Soreness, DOMS). Die Rate der Creatin-Kinase ist klinisch von der $C W I$ beeinflusst. Bezüglich der Funktionsfähigkeit der unteren Extremitäten gab es keinen statistisch signifikanten Unterschied zwischen $C W I$ oder $C W T$. Alle diese Ergebnisse sind gültig für 24 Stunden nach der Übung.

Schlussfolgerung: Im Allgemeinen wurden Verbesserungen nach der Anwendung von $C W T$ oder $C W I$ beobachtet. Letztere zeigt sich besonders wirksam bei der Verringerung der DOMS bis 24 Stunden. Allerdings ist die analysierte Stichprobe zu klein und weist einige Verzerrungen auf. Weitere Studien sind erforderlich, um den Nutzen der Immersion als Regenerierungstherapie zu bewerten.

Stichwörter: Kaltwasserimmersion, Kalt/Warm-Behandlung, Regenerierung, Sport, Muskelkater, Creatin-Kinase, funktionelle Kapazität 


\section{Remerciements}

Nous tenons à adresser nos sincères remerciements à :

Monsieur Nicolas Mathieu, directeur de notre travail de Bachelor et chargé de cours à la HES-SO Valais, pour sa disponibilité, ses remarques pertinentes et ses nombreux conseils.

Monsieur Roger Hilfiker, professeur à la HES-SO Valais, pour son aide et ses précisions en matière de statistiques.

Monsieur Matthieu Emery, professeur de français et d'éducation physique, pour la relecture et la correction de notre travail.

Madame Maria Rompf, $P h D$, doctorat en Sciences de la Vie, pour la correction du résumé en allemand.

Monsieur François Bieuzen, $P h D$, doctorat en Sciences du Mouvement Humain et chercheur au laboratoire Sport, Expertise et Performance (INSEP), pour les informations échangées et les articles reçus.

Tous les auteurs, qui nous ont envoyé leurs études et leurs résultats.

Monsieur Pierre Humbert-Droz, pour l'impression finale de notre travail.

Nos proches, pour la participation indirecte et le soutien apporté tout au long de ce projet. 


\section{Table des matières}

1. Introduction .............................................................................................................................................. 1

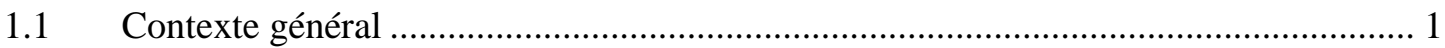

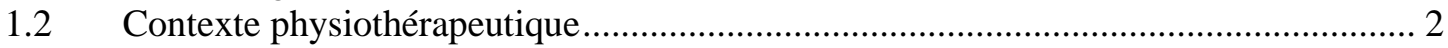

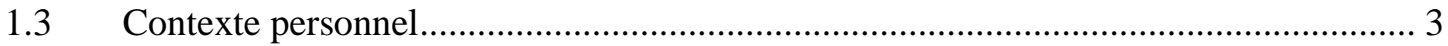

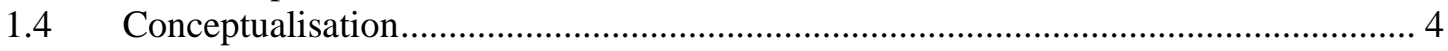

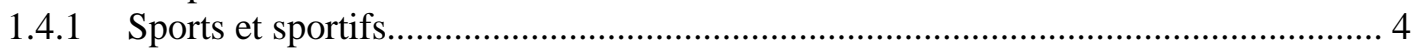

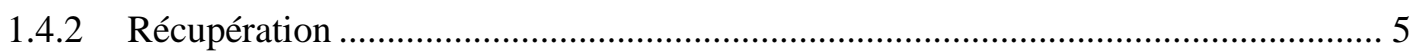

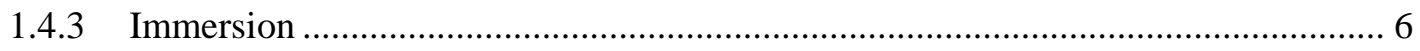

1.4.3.1 Effets physiologiques de l'immersion............................................................. 7

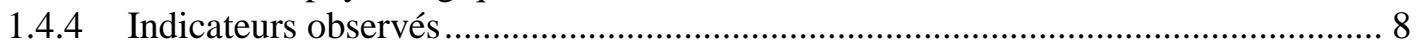

1.4.4.1 Douleurs musculaires d'apparition retardée $(D O M S)$...................................... 9

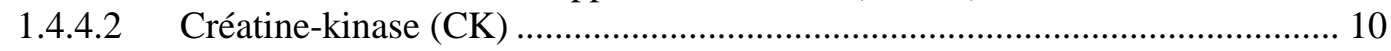

1.4.4.3 Capacité fonctionnelle des membres inférieurs ......................................... 13





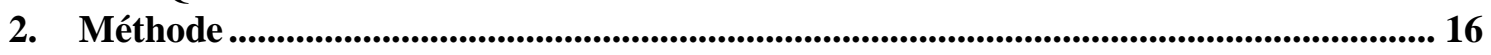



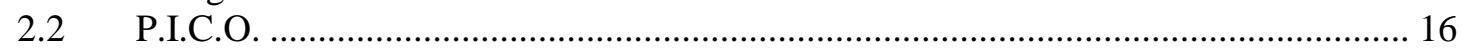

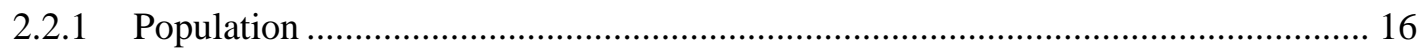

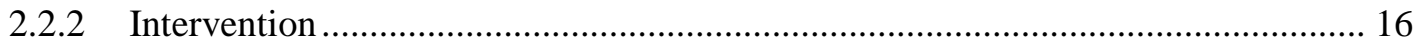

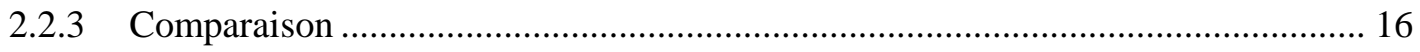

2.2.4 Outcomes

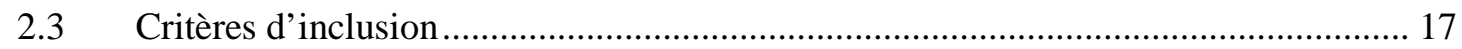

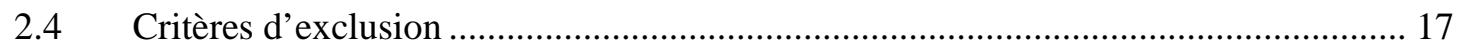

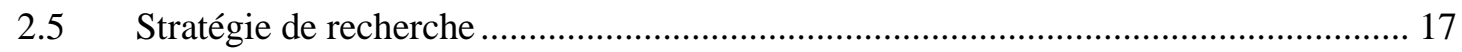

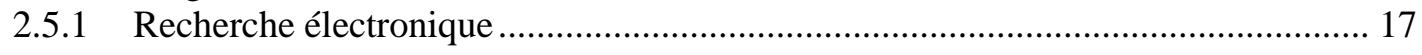

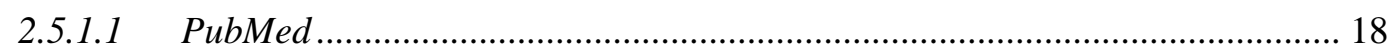

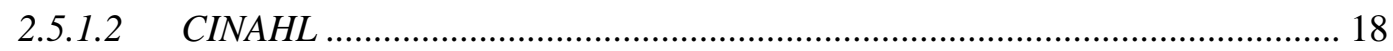

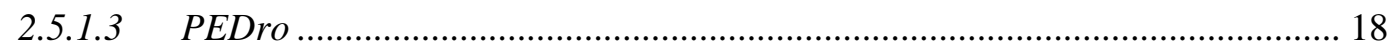

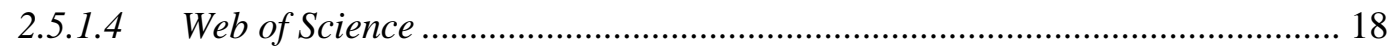

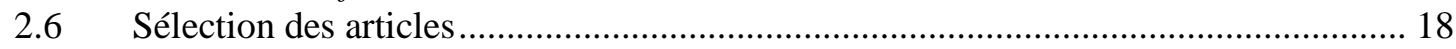

2.6.1 $1^{\mathrm{er}}$ tri : sélection par titres, résumés et doublons .................................................. 18

2.6.2 $2^{\text {ème }}$ tri : sélection par lecture de la version intégrale ........................................... 19

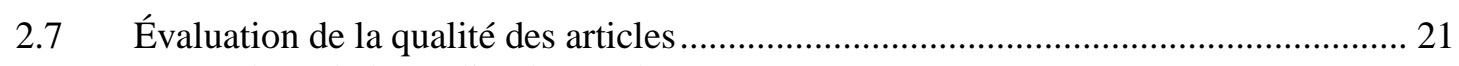

2.7.1 Résultats de la qualité des études...................................................................... 21

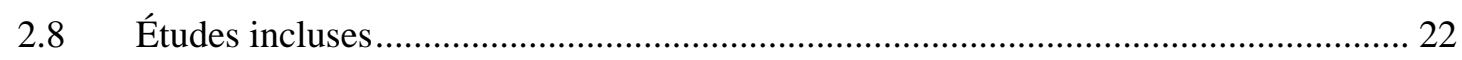

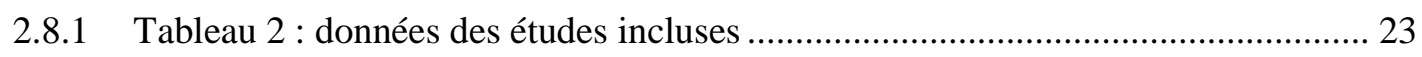

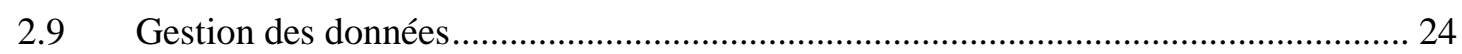



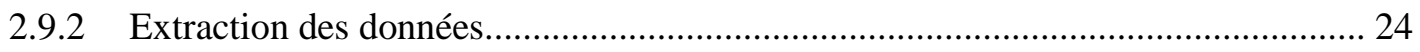



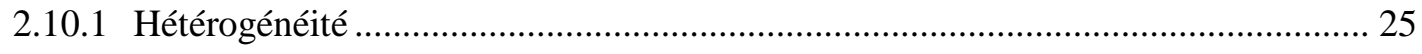

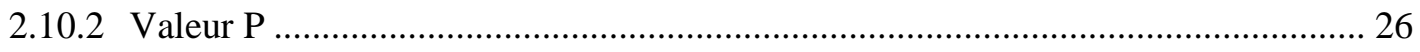




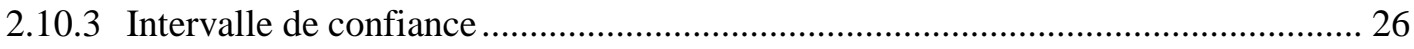

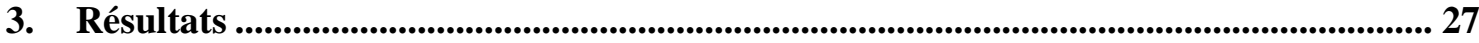

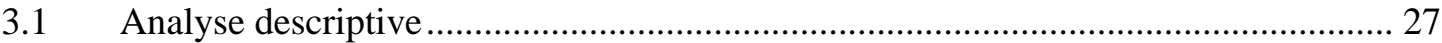

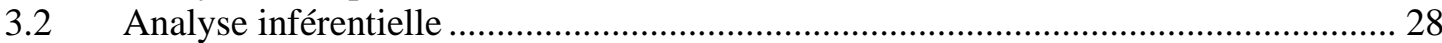

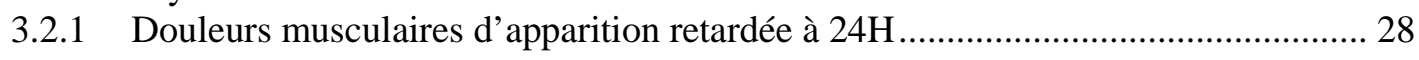

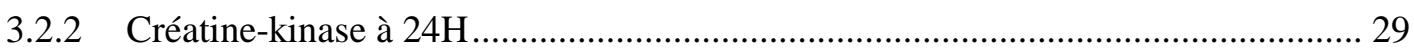

3.2.3 Capacité fonctionnelle des membres inférieurs à $24 \mathrm{H}$............................................ 30

3.2.4 Récapitulatif des résultats des forest plots ............................................................. 31

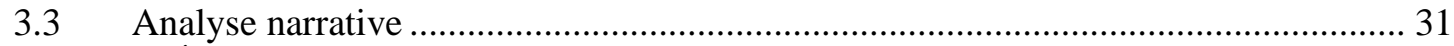

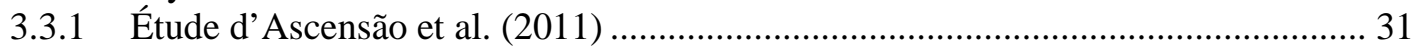

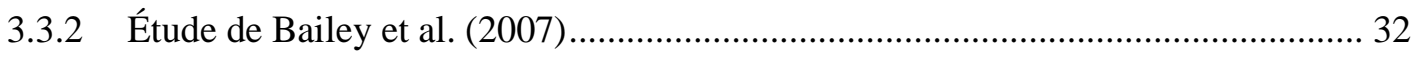

3.3.3 Étude de Pournot et al. (2011) ............................................................................ 32

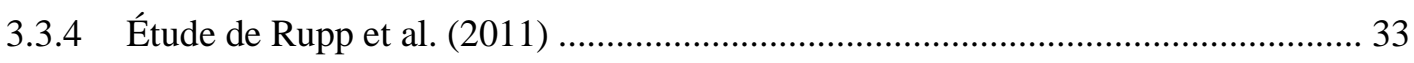

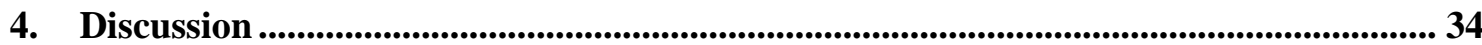

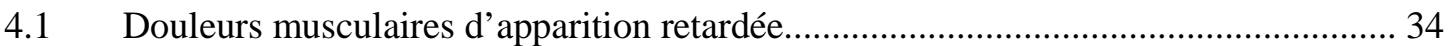

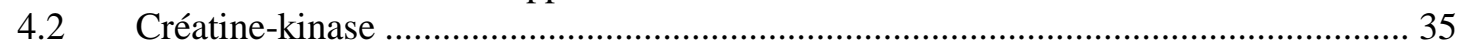

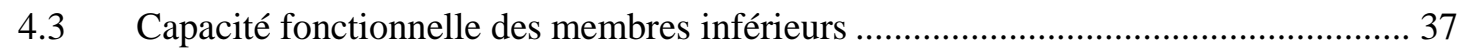

4.4 Autres paramètres observés dans la littérature......................................................... 38

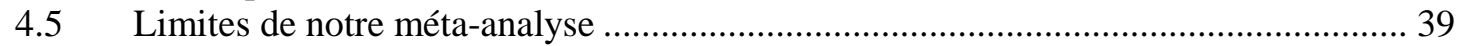

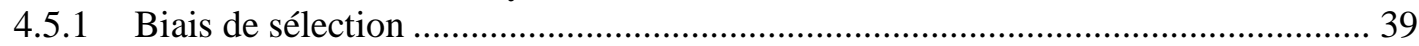

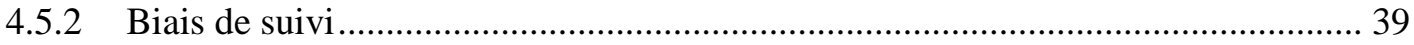

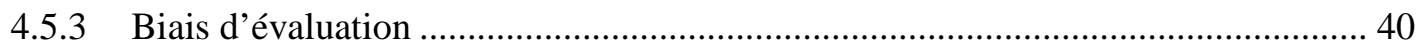

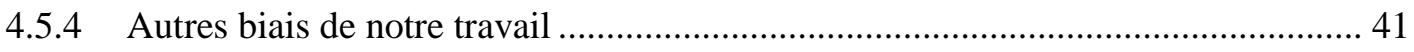

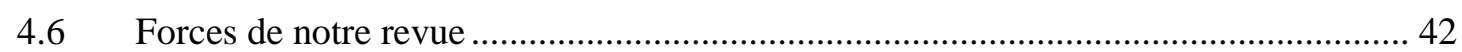

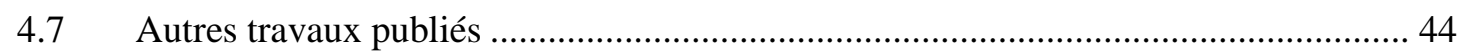

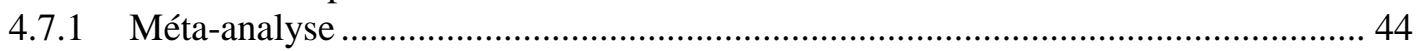

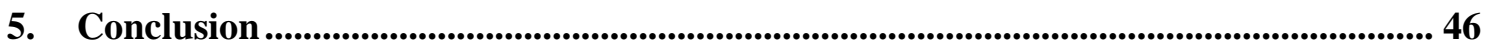

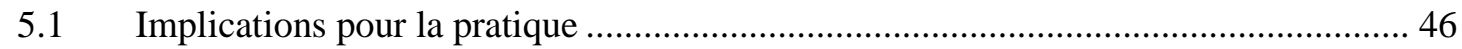

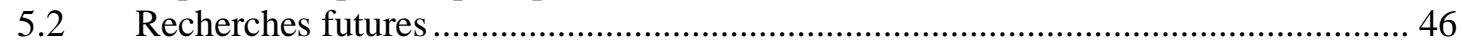

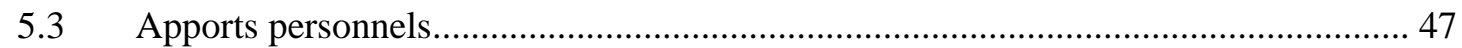

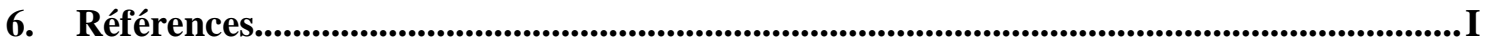

7. Liste des illustrations et des tableaux..........................................................................................XI

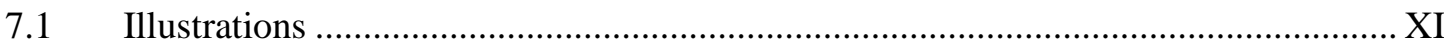

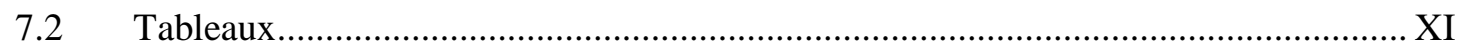

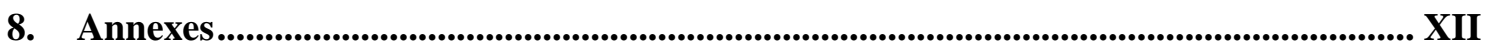

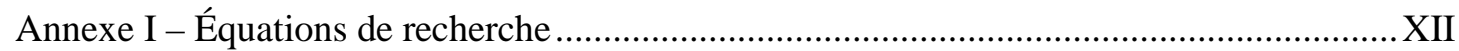

Annexe II - Échelle PEDro.............................................................................................

Annexe III - Tableaux d'extraction des données ........................................................... XIV

Annexe IV - Forest plots non pris en compte................................................................. XVI 


\section{Introduction}

\subsection{Contexte général}

Depuis les années 1990-2000, les physiologistes, les entraineurs, les préparateurs physiques et les physiothérapeutes du sport s'intéressent fortement à l'immersion en eau, corps entier ou segmentaire, en vue d'optimiser la récupération musculaire posteffort des sportifs (Hausswirth et al., 2010) (Vaile et al., 2008).

Les athlètes s'entraînent en utilisant des charges conséquentes de travail dans le but de perfectionner leur performance physique (De Nardi et al., 2011). Cependant, des charges excessives lors des entraînements et des compétitions, particulièrement avec des temps minimes de récupération, amènent des contraintes sur le système musculosquelettique, provoquent des symptômes de surcharge de fatigue et diminuent la performance du sportif (Reilly \& Ekblom, 2005). Afin de maintenir cette dernière, les entraîneurs et les athlètes utilisent des méthodes post-exercices de régénération musculaire. Ils ont ainsi besoin de maximiser les modalités et les périodes de récupération afin de réduire le temps de récupération (Pournot et al., 2011) (Ingram et al., 2009).

De nos jours, il existe une grande variété de méthodes de récupération comme la récupération active, le massage, l'électrothérapie, la thérapie par oxygène hyperbarique, les médicaments anti-inflammatoires non-stéroïdiens, les vêtements de compression, le stretching ou encore l'immersion, qu'elle soit en eau froide ou en eau contrastée (Barnett, 2006). Certaines méthodes, dont la récupération active, ont clairement démontré leurs impacts positifs sur la récupération (Signorile et al., 1993) alors que d'autres, sont utilisées de manière courante dans le milieu sportif mais ne montrent pas de consensus dans la littérature scientifique. C'est le cas, notamment, pour la thérapie par immersion. Plusieurs études mentionnent des effets positifs de celle-ci sur la récupération (Bailey et al., 2007) (Lane \& Wenger, 2004), tandis que d'autres la mettent en doute (Sellwood et al., 2007) (Yamane et al., 2006). Or, une étude de Van Wyk \& Lambert (2009) démontre que 96\% des professionnels de la santé en Nouvelle-Zélande utilisent l'immersion en eau froide après un match de rugby. 
Aucune modalité de l'immersion, à savoir, la durée d'immersion, le niveau corporel immergé et la température utilisée, n'est clairement établie. Il est donc intéressant d'analyser la littérature scientifique afin d'obtenir de meilleures connaissances sur les effets de cette technique, dans le but de conseiller les sportifs de manière optimale.

\subsection{Contexte physiothérapeutique}

La physiothérapie du sport est une discipline qui fait partie intégrante de la médecine du sport. Son importance grandissante dans le sport de haut niveau et amateur est principalement liée aux exigences et aux contraintes du sport moderne et de la compétition (Commission médicale du Comité Internationale Olympique, 2008).

L'activité du physiothérapeute du sport consiste, entre autres, à la réhabilitation des blessures aiguës ou chroniques du sportif. La prise en charge d'un tel sportif s'effectue jusqu'au recouvrement totale des capacités fonctionnelles permettant une pratique de son sport de manière adéquate et optimale. Le physiothérapeute a également un rôle extrêmement important dans la prévention des blessures en travaillant sur les qualités physiques du sportif et en corrigeant les déficits que son activité peut engendrer. Il est présent lors de la préparation précompétitive afin de mettre l'athlète dans les meilleures conditions possibles en lui prodiguant des soins adaptés. Lorsqu'un mécanisme lésionnel se produit, le physiothérapeute du sport intervient sur le terrain en prodiguant les premiers soins.

Finalement, il s'occupe de la phase de récupération post-effort du sportif afin de diminuer la fatigue, d'accélérer le taux de régénération physiologique, de faciliter l'augmentation des charges et de diminuer les risques de blessure (Bompa \& Haff, 2009). Cela permet ainsi à l'athlète de retrouver plus rapidement ses capacités physiques optimales. Afin d'atteindre ces buts, il existe différents moyens thérapeutiques de récupération comme précédemment cités. Néanmoins, certains sont plus utilisés que d'autres dans le milieu sportif malgré le manque d'évidence scientifique (Kellmann, 2010) (Vaile et al., 2008). Beaucoup d'athlètes et d'entraîneurs utilisent aujourd'hui l'immersion en eau contrastée (froid/chaud) comme une stratégie post-exercice avec l'intention d'améliorer la récupération (Coffey et al., 2004). 
Par exemple, dans le rugby, les modalités de récupération les plus utilisées sont le stretching et l'immersion en eau froide, suivies par la récupération active et le massage (Van Wyk \& Lambert, 2009).

Il est important pour le physiothérapeute du sport d'être à la pointe des connaissances actuelles en matière de récupération dans le but d'optimiser la prise en charge posteffort de l'athlète, tant par des techniques actives ou passives, que par des conseils spécifiques.

\subsection{Contexte personnel}

De part nos études en physiothérapie, les sportifs de notre entourage nous questionnent souvent sur les différents moyens utilisés dans la récupération. Lorsque leurs questions deviennent très spécifiques, nous rencontrons des difficultés dans les réponses à formuler.

En effet, suite à notre cursus en physiothérapie, nous avons abordé de manière succincte la récupération post-effort, mais également les différentes techniques de thermothérapie. Nous avons alors appris que le froid pouvait permettre une meilleure récupération physique. Cependant ces notions sont restées superficielles et ne nous permettent pas de répondre à nos proches ainsi qu'à nos collègues issus de différents milieux sportifs. Cette situation est pour nous frustrante. De ce fait, travailler sur une modalité de récupération nous permettra certainement d'être plus apte à prodiguer des conseils appropriés.

De plus, nous pratiquons différents sports à un niveau amateur et portons donc un fort intérêt pour la physiothérapie du sport. Il nous semble judicieux d'approfondir ce thème afin d'acquérir les connaissances adéquates pour aborder dans de meilleures conditions notre future profession et ainsi apporter des explications plus précises à nos patients sportifs mais également à notre entourage. 


\subsection{Conceptualisation}

\subsubsection{Sports et sportifs}

Dans la société actuelle, le sport est devenu une activité physique courante, que ce soit par simple plaisir, pour le maintien d'une condition physique ou pour la performance quelle qu'elle soit. Le sport est défini, selon l'encyclopédie Larousse (2009), comme un «ensemble d'exercices physiques se présentant sous forme de jeux individuels ou collectifs, donnant généralement lieu à compétition, pratiqués en observant certaines règles précises ». Le sport est un terme général regroupant un nombre varié d'activités qui demande des capacités physiques et mentales appropriées, aussi bien dans les sports individuels que collectifs. D'autres termes sont également utilisés en lieu et place du sport, notamment l'exercice et l'activité physique. Cette dernière est décrite comme «tout mouvement corporel produit par les muscles squelettiques qui entraine une dépense d'énergie » et l'exercice, qui est un composant de l'activité physique, comme « un mouvement corporel planifié, structuré et répétitif fait pour améliorer ou maintenir un ou plusieurs composants de la condition physique » (Caspersen et al., 1985).

Plus précisément, selon Biddle \& Mutrie (2001), le sport est «un sous-composant de l'exercice où l'activité, structurée et compétitive, est régie par des règles et implique un mouvement moteur caractérisé par la stratégie physique, la prouesse et la chance ».

La pratique des sports peut être divisée en trois catégories : les sports cycliques, les sports acycliques et les sports acycliques combinés. Un sport cyclique est caractérisé par un seul mouvement répété continuellement (ex : course à pied, cyclisme, etc.). Un sport acyclique requiert pour chaque action différents types de mouvement, plutôt qu'un même mouvement répété dans un cycle (ex : football, tennis, etc.). Un sport acyclique combiné alterne des activités cycliques et acycliques (ex : 3000m steeple, biathlon, etc.) (Thompson, 2011).

Chaque type de sport a besoin d'un entraînement spécifique en fonction des qualités requises et des contraintes imposées. Cela demande de respecter les principes d'entraînement propre à l'activité physique exercée ainsi que des temps programmés de repos. En effet, un marathonien aura un temps de récupération plus long qu'un footballeur suite à une compétition car le type d'effort n'est pas le même. 
Dans l'antiquité, l'athlète était celui qui disputait des concours organisés dans le cadre des fêtes et des jeux publics. Aujourd'hui, l'athlète ou le sportif est défini tout simplement comme une personne pratiquant un sport (Larousse, 2009). De plus, il existe des différences marquées entre les sportifs occasionnels, les amateurs et les professionnels, tant au niveau des objectifs, du nombre d'entraînements, que de l'intensité de ceux-ci.

Selon Rochcongar (2009), le sportif amateur s'entraîne au maximum trois fois par semaine. De plus, selon nous, il pratiquera cette activité par plaisir. A contrario, le professionnel s'entraînera dans le but d'atteindre les meilleures performances possibles tout en percevant une rémunération variable en fonction du niveau dans lequel il évolue et lui permettant de subvenir à ses besoins quotidiens.

Que ce soit dans le milieu amateur ou professionnel, la récupération musculaire est un facteur clé de la performance car elle joue un rôle primordial dans la qualité et l'enchaînement des entraînements (Hausswirth et al., 2010).

\subsubsection{Récupération}

Pour définir la récupération, nous devons en amont définir la fatigue musculaire, car ces deux notions sont dépendantes. En effet, la récupération n'est pas nécessaire sans une fatigue préalable quelle qu'elle soit. La fatigue musculaire regroupe l'ensemble des phénomènes limitant la poursuite d'une activité motrice ou retardant la restauration de la capacité de travail en un temps normal. Elle a été décrite par Scherrer \& Monod (1960) comme « une baisse transitoire de la capacité de travail du muscle, consécutive à l'activité musculaire, survenant pour un niveau constant d'incitation des centres moteurs et réversible par le repos ». Cela vaut pour le muscle mais également pour l'organisme entier comme l'évoquent Monod et al. (2011).

Pour Kellmann \& Kallus (2001), la récuperation est « un processus actif inter- et intraindividuelle de niveau multiples (psychologique, physiologique, social) qui se poursuit dans le temps pour le rétablissement des capacités de performance ». Une autre définition nous paraît intéressante. Selon le Dr. Guezennec, la période de récupération correspond «au temps nécessaire pour que les différents paramètres physiologiques modifiés par l'exercice rejoignent les valeurs de l'état au repos ». 
La récupération est donc dépendante de l'effort, de la durée et de l'intensité de l'exercice. Elle se déclenche dès le démarrage de l'activité musculaire. Si l'effort est faible (par exemple lors de la marche), les processus de resynthèse et ceux de dégradation s'équilibrent. Si l'effort est de très grande intensité, les processus de dégradation «noient » ceux de resynthèse et l'effort cesse rapidement. Dès que l'effort s'arrête, les processus de resynthèse prédominent sur ceux de dégradation.

Il y a deux phases dans la récupération : une phase rapide directement après l'effort et une phase lente, plusieurs heures après l'exercice. Plus l'athlète se fatigue en créant un déséquilibre dans son organisme, plus la récupération sera importante pour retrouver un nouvel équilibre. La récupération totale de l'exercice musculaire est un phénomène irrégulier dans le temps et sa durée est tributaire de la restauration des réserves métaboliques. C'est pourquoi, une attention particulière est apportée aux différentes recherches et à l'application de techniques de récupération afin de l'optimiser (Leeder et al., 2012).

\subsubsection{Immersion}

La littérature scientifique relate principalement quatre modalités d'immersion corps entier ou segmentaire, en fonction de la température :

- en eau tempérée (de $15^{\circ}$ à $36^{\circ}$ Celsius),

- en eau chaude $\left(42^{\circ} \mathrm{C}\right)$,

- en eau froide $\left(<15^{\circ} \mathrm{C}\right)$

- et en eau contrastée (alternance en eau froide entre $8^{\circ}$ et $15^{\circ} \mathrm{C}$ et en eau chaude entre $38^{\circ}$ et $45^{\circ} \mathrm{C}$ ) (C. Bleakley et al., 2012) (Low et al., 2000).

Dans les différentes études, nous retrouvons les termes anglais suivants : Cold Water Immersion $(C W I)$ qui se rapporte à l'immersion en eau froide, Contrast Water Therapy $(C W T)$ qui est égal à l'immersion en eau contrastée, Thermoneutral Water Immersion (TWI) pour l'immersion en eau tempérée et la Hot Water Immersion (HWI) qui fait référence à l'immersion en eau chaude.

Le patient peut être immergé corps entier (jusqu'à hauteur de la colonne cervicale) (Vaile et al., 2011), jusqu' aux épaules (Malkinson et al., 1981), au sternum (Mantoni et al., 2007) ou partiellement, à hauteur des crêtes iliaques (Ascensão et al., 2011). 
Dernièrement, la $C W I$ est devenue une des interventions des plus populaires afin de prévenir les courbatures et pour améliorer la récupération post-exercice (Bleakley et al., 2012). Elle a tout d'abord été utilisée par les sportifs d'élite et s'est démocratisée par la suite. Directement après l'effort, les sportifs s'immergent dans des bains froids. Les protocoles d'immersion varient que ce soit pour la température de l'eau, le temps d'immersion ou encore le volume de corps immergé (Bleakley et al., 2012).

\subsubsection{Effets physiologiques de l'immersion}

Les effets physiologiques de l'immersion sont liés à la température de l'eau et à la pression (Leeder et al., 2012) qui induisent des changements dans le flux sanguin et la température musculaire (Gregson et al., 2011) (Bleakley \& Davison, 2010).

La $C W I$, de part l'effet du froid, induit une diminution de la température de la peau, de la couche sous-cutanée et du muscle (Cochrane, 2004). La diminution de la température des tissus stimulerait les récepteurs cutanés entraînant les fibres sympathiques à effectuer de manière indirecte une vasoconstriction. Cela diminuerait le gonflement et l'inflammation par le ralentissement du métabolisme et de la production de métabolites (Cochrane, 2004). Cette vasoconstriction locale induit une réduction de la perméabilité cellulaire, lymphatique et capillaire, qui a pour conséquence, une plus basse concentration de créatine-kinase (Eston \& Peters, 1999). L'immersion en eau froide stimule le retour veineux et aide à éliminer les métabolites après un exercice, réduisant la fatigue musculaire et la réponse inflammatoire (Bleakley \& Davison, 2010) (Hausswirth et al., 2010). Cette dernière peut diminuer la douleur, le gonflement et la perte de la force musculaire (Goodall \& Howatson, 2008) (Smith, 1991). La CWI altère la transmission nerveuse et diminue la vitesse de conduction nerveuse dans les tissus superficiels ralentissant la fréquence des afférences au niveau du fuseau musculaire et de la réponse réflexe, diminuant ainsi les spasmes musculaires et la douleur (Cochrane, 2004).

Dans une étude sur les réponses physiologiques liées à une immersion en eau froide, Hausswirth et al. (2010) explique qu'un deuxième mécanisme est induit par l'immersion, c'est la pression hydrostatique sur la ou les parties du corps immergées. En effet, la pression de l'eau exerce une pression supérieure à celle de l'air sur le corps provoquant un mouvement des gaz, substances et fluides. Cela permettrait de réduire la 
taille de l'œdème produit par l'exercice mais également de limiter l'influx nerveux par compression des muscles et des nerfs. Les adaptations physiologiques liées à ce phénomène peuvent varier en fonction des études car elles n'appliquent pas toutes les mêmes procédures d'immersion et génèrent donc plus ou moins de pression sur le corps (Hausswirth et al., 2010).

La $H W I$ augmente la température des tissus, le flux sanguin local, l'élasticité musculaire et provoque une vasodilatation locale. Elle augmente également la production de métabolites et réduit les spasmes musculaires (Cochrane, 2004) (Brukner \& Khan, 2001) (Prentice, 1999) (Zuluaga, 1995). Le réchauffement superficiel diminue la conduction nerveuse sympathique, ce qui provoque une vasodilatation locale des vaisseaux sanguins et augmente la circulation. Cette dernière permet un apport accru en oxygène, en anticorps et augmente la possibilité d'évacuer les métabolites (Cochrane, 2004) (Zuluaga, 1995).

La $C W T$ génère successivement des vasoconstrictions et des vasodilatations ayant pour effet de stimuler le flux sanguin tout en réduisant la réponse inflammatoire ainsi que sa durée. Ce phénomène de pompage vasculaire permettrait le mouvement des substances métaboliques, la réparation du muscle soumis à l'exercice et la diminution des processus métaboliques au sein du muscle (Cochrane, 2004). Ces mouvements augmenteraient notamment le transfert des métabolites vers le sang et réduirait le volume du liquide intracellulaire (Hausswirth et al., 2010).

Il y a un manque d'investigations et d'évidences scientifiques afin d'expliquer les bénéfices potentiels et/ou les mécanismes physiologiques des différents types d'immersion (Vaile et al., 2008). Afin de juger de l'efficacité des différentes techniques d'immersion, il est important de les évaluer grâce à certains indicateurs spécifiques au sport.

\subsubsection{Indicateurs observés}

Nous avons sélectionné trois indicateurs afin d'évaluer les effets des différentes modalités d'immersion. Ceux-ci sont les douleurs musculaires d'apparition retardée pour le ressenti subjectif, la créatine-kinase pour l'évaluation biologique et la hauteur de saut vertical pour la capacité fonctionnelle des membres inférieurs. 


\subsubsection{Douleurs musculaires d'apparition retardée (DOMS)}

DOMS est l'abréviation anglaise de Delayed Onset Muscle Soreness qui signifie littéralement : douleurs musculaires d'apparition retardée. En français, elles sont plus communément appelées courbatures et apparaissent de manière fréquente lors de la pratique du sport (Coudreuse et al., 2004).

Les courbatures constituent un véritable syndrome musculaire inflammatoire post-effort (SMIPE), résultant d'un travail excentrique effectué à haute intensité. Elles sont à l'origine de cinq dysfonctionnements principaux (Coudreuse et al., 2004) :

- une douleur diffuse majorée par le travail excentrique apparaissant 12 à 48 heures après un effort musculaire intense plus ou moins à prédominance excentrique ;

- une diminution de la force musculaire (environ $40 \%$ );

- une diminution de la proprioception ;

- une diminution des amplitudes articulaires ;

- un œdème de faible importance.

Selon Armstrong et al. (1991), le mécanisme inflammatoire comprend trois stades :

- le $1^{\text {er }}$ stade (auto-génique) se déroule durant les trois premières heures après l'exercice et correspond au début de la dégradation des structures membranaires lésées.

- le $2^{\text {ème }}$ stade (phagocytaire) : la lésion est envahie par de nombreux monocytes qui se transforment en phagocytose. On observe une progression de la pression et de la température interne du muscle qui augmentent la décharge spontanée des nocicepteurs de type III et IV. Celles-ci sont issues de terminaisons nerveuses libres, situées dans l'ensemble des tissus musculaires et conjonctifs, en particulier à la jonction tendineuse (Kniffki et al., 1978). Une fois stimulées, ces terminaisons nerveuses libres situées au niveau des tissus lésés libèrent de la substance $\mathrm{P}$, ce qui aboutit à une amplification de la réponse inflammatoire. Cela favorise donc les nocicepteurs et l'hyperalgie globale propre à la courbature.

- le $3^{\text {ème }}$ stade (régénération) débute après 4 à 6 jours (Faulkner et al., 1993).

Selon le type et l'intensité de l'exercice, les douleurs peuvent durer généralement entre 2 à 5 jours et les déficits persistent le plus souvent de 2 à 10 jours après l'apparition des 
douleurs, correspondant à une phase d'inhibition du muscle face aux contraintes, durant la période où perdurent les microlésions (Coudreuse et al., 2004).

Dans les études scientifiques, la mesure des douleurs ressenties se fait grâce à l'utilisation d'une échelle visuelle analogue (EVA). Celle-ci peut varier dans les limites inférieures et supérieures. Dans leur étude, Sellwood et al. (2007) mesurent les douleurs sur une échelle comprise entre 0 et $100 \mathrm{~mm}$. Un score de 0 signifie pas de douleur alors que 100 représente la pire douleur possible, Pournot et al. (2011) utilisent quant à eux une échelle entre 0 et 10 avec comme limite inférieure 0 (normal) et comme limite supérieure 10 (extrêmement douloureux). De plus, la prise des mesures de la douleur peut être effectuée à des temps pré- et post-effort variables en fonction du sport pratiqué et des auteurs (Pournot et al., 2011) (Sellwood et al., 2007).

\subsubsection{Créatine-kinase (CK)}

La créatine-kinase, également appelée créatine phosphokinase (CPK) est une enzyme qui se trouve au niveau musculaire mais aussi sous deux autres formes : une spécifique dans le cour et une dans le cerveau.

Lors de la contraction musculaire, l'énergie est fournie par l'adénosine-tri-phosphate (ATP). Les quantités d'ATP en réserve dans le muscle étant faibles (ne permettent qu'une contraction de 4 à 6 secondes), le stock d'ATP doit être constamment renouvelé au fur et à mesure qu'il est utilisé afin de permettre la poursuite de la contraction musculaire. Lors de celle-ci, l'ATP est hydrolysé en adénosine-di-phosphate (ADP) et en phosphate inorganique.

La régénération de l'ATP s'effectue en une fraction de seconde grâce aux trois voies suivantes :

- par la phosphorylation de l'ADP par la créatine-phosphate et l'action de la CK

- Par le mécanisme anaérobie : glycolyse et production d'acide lactique

- Par la respiration cellulaire aérobie

Nous allons nous concentrer uniquement sur le mécanisme de phosphorylation étant donné que les études observent dans la plupart des cas le niveau de CK comme 
marqueur de fatigue musculaire (Ingram et al., 2009) (Vaile et al., 2008) (Bailey et al., 2007).

La CK a un rôle de catalyseur dans la conversion de l'ADP en ATP et inversement. Par l'action de la $\mathrm{CK}$, la créatine-phosphate est hydrolysée, ce qui libère un groupement phosphate et de l'énergie vers l'ADP qui devient de l'ATP. La réaction est réversible, de sorte que lorsque le muscle est riche en ATP, l'énergie est récupérée pour faire de la créatine-phosphate. Au contraire, lorsque l'ATP redevient nécessaire, ce transfert direct restitue l'énergie mise en réserve. Cette réaction permet de mettre en réserve de l'ATP et de le libérer lors de la contraction musculaire. Cela n'entraîne aucun phénomène d'oxydation et n'engendre pas d'acide lactique (Marieb et al., 2010) (Faculté de Médecine Pierre et Marie Curie).

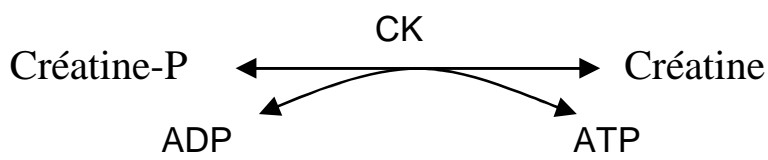

Figure 1 : transformation de l'ADP en ATP par l'action de la CK sur la Créatine-P ${ }^{1}$

Lorsque l'intensité de l'exercice est légère à moyenne, le tissu musculaire ne présente pas de changement dans sa perméabilité membranaire. A contrario, si l'intensité devient élevée, la perméabilité membranaire se modifie et la CK est relâchée dans le liquide interstitiel. Elle se mélange ensuite au système lymphatique et finit dans la circulation sanguine. (Brancaccio et al., 2007). L'apparition de la CK dans le plasma est un marqueur de dommages musculaires dans le sport (Hortobágyi \& Denahan, 1989).

Le niveau de CK dans le plasma dépend de l'âge, du sexe, du genre, de la masse musculaire, de l'activité physique et des conditions climatiques (Brancaccio et al., 2007). Les jeunes adultes ont un taux de sérum de CK élevé (Borges \& EssénGustavsson, 1989) qui diminuera progressivement au fil des années (Tietz, Shuey, \& Wekstein, 1992). Les femmes ont un taux de CK plus faible que les hommes. Cela peut s'expliquer par une quantité d'œstrogène plus élevée chez la femme qui influence la perméabilité membranaire. Les caucasiens ont des valeurs de $\mathrm{CK}$ inférieurs à celles des hommes de type africain. Les sportifs ayant un diamètre et un volume du quadriceps

1 Biochimie et nutrition des activités physiques et sportives: Le métabolisme énergétique. Masson. (Pilardeau, 1995) 
élevés ont une augmentation plus importante de la CK. Le type d'exercice ainsi que sa durée influence la variation de CK post-exercice. Les sports de combat (intensité élevée et de durée courte et répétée) ou les sports d'endurance comme le marathon ou le triathlon (intensité modérée de longue durée), ainsi que les efforts de type excentrique présentent des taux élevés de CK (Brancaccio et al., 2007) (Pilardeau, 1995). Une température froide pendant l'exercice provoque une plus forte augmentation de la CK par rapport au même exercice effectuée dans des conditions climatiques chaudes.

Les moyennes du taux de CK plasmatique au repos chez l'homme sont comprises entre 35-175 Unités/Litre (U/L) (Gagliano et al., 2009), avec des différences de 20 à 16’000 U/L. De telles variations ont lieu à cause de certains troubles cliniques, de blessures légères, de facteurs génétiques, de l'activité physique ou encore de médicaments (Prelle et al., 2002). Dans les exemples de rhabdomyolyse (lésion/destruction musculaire diagnostiquée cliniquement), les taux de CK peuvent se situer entre 10'000-200'000 U/L et voire même jusqu'à 3 x 10 $10^{6}$ U/L dans des cas extrêmes (Efstratiadis et al., 2007). De tels niveaux signalent clairement une forte perturbation ou la désintégration du tissu musculaire strié et la fuite des constituants intracellulaires musculaires dans la circulation sanguine. En l'absence d'infarctus du myocarde ou du cerveau, de traumatismes physiques ou de maladies, les taux sériques de CK supérieurs à $5000 \mathrm{U} / \mathrm{L}$ sont un indicateur de perturbations graves des muscles (Baird et al. 2012) (HuertaAlardín et al., 2005).

Lors d'un exercice à charges excentriques ou dans des activités similaires comme la course en descente, la $\mathrm{CK}$ peut augmenter de 10 à 20 fois par rapport à la valeur normale (Latham et al., 2008). Dans une étude de Takarada (2003), les valeurs de CK avant un match de rugby étaient de $242( \pm 125)$ U/L et ont atteint un maximum après la compétition de 1047 ( \pm 156) U/L. Gill et al. (2006) ont également étudié les valeurs précompétitives et post-compétitives moyennes lors d'un match de rugby. Ces valeurs étaient de 1023 ( \pm 308.3) U/L avant la compétition et de $2194.0( \pm 833.7)$ U/L après celle-ci. De part la variabilité des valeurs retrouvées dans la littérature scientifique, il n'existe pas de norme universellement acceptée quant à l'augmentation du taux de CK lors d'une activité physique (Baird et al., 2012). 
Selon l'article de Jastrzębski (2006), l'élévation maximale du taux de CK dans le sang est atteinte dans les 18 à 24 heures post-effort. En fonction de l'intensité et de la durée de l'activité physique, la valeur de CK diminue progressivement pour retrouver sa valeur de base dans les 2 à 10 jours (Brancaccio et al., 2007) (Takarada, 2003) (Ehlers et al., 2002).

\subsubsection{Capacité fonctionnelle des membres inférieurs}

L'évaluation des capacités de l'athlète est une des plus grandes questions du sport moderne. De nombreux tests sont utilisés dans des procédures de sélections, afin d'obtenir un screening des sujets ou encore pour évaluer l'efficacité d'un entraînement (Norkowski, 2002).

La puissance est définie comme la force appliquée multipliée par la vitesse de mouvement (Howard G. Knuttgen, 1987). La puissance explosive est le principal déterminant de la performance dans les activités requérant une séquence de mouvement effectuée à haute vitesse. Les actions musculaires explosives sont nécessaires pour frapper, sauter et lancer ainsi que lors des accélérations et des changements de direction dans différents sports. (Newton \& Kraemer, 1994). Le saut vertical propose une mesure effective de la puissance et donc une mesure importante et indirecte de la performance (Buckthorpe et al., 2012) (Klavora, 2000) (Newton \& Kraemer, 1994). De plus, Wisloff et al. (2004) ont démontré, par exemple, qu'il existe une forte relation entre le saut vertical et les sprints chez les footballeurs.

Il existe trois modalités différentes de saut :

- le squat jump (SJ),

- le contre-mouvement jump (CMJ)

- et le drop jump (DJ).

Le $S J$ tente de mesurer la détente «pure», sans étirement musculaire préalable. La position de départ se fait avec une flexion de genou de $90^{\circ}$ et les mains sur les hanches. À partir de cette position, le sujet effectue un saut avec une poussée maximale vers le haut (Cometti, 1997). 
Le $C M J$ se fait dans les mêmes conditions que le $S J$ à la différence près que le sujet aura une position de départ différente. Le sujet commence le test en position debout bipodale avec les membres inférieurs tendus, effectue un mouvement rapide de flexion des genoux puis réalise un saut vers le haut. Cette modalité en fait donc un test pliométrique (cycle étirement-raccourcissement) (Cometti, 1997).

Le $D J$ a la même position de départ que le $C M J$ mais le sujet va démarrer le test d'un élément surélevé $(20 \mathrm{~cm}, 40 \mathrm{~cm}, 60 \mathrm{~cm}$ ou $80 \mathrm{~cm}$ ) en se laissant tomber, et effectuer un « saut-rebond » vertical (Cometti, 1997).

Selon Malliou et al. (2003), le saut vertical peut être testé soit en laboratoire soit sur le terrain. Les matériels de test de saut sont très variés :

- les tapis de contact qui calculent la hauteur du saut par rapport au temps de vol $\left(\right.$ Bosco $\left.^{\circledR}\right)$,

- des systèmes de ceintures raccrochés par un fil à un instrument au sol qui, grâce à ce fil, calculent la hauteur du saut (Dynabiopsy $\left.{ }^{\circledR}\right)$,

- des bases au sol qui calculent la hauteur du saut grâce à la force de réaction au sol exercée sur la base lors de l'impulsion (Buckthorpe et al., 2012)

- et grâce au Myotest ${ }^{\circledR}$, qui est un appareil portatif utilisant l'accélérométrie et validé pour l'évaluation de divers paramètres de la performance (Castagna et al., 2013) (Crewther et al., 2010).

La hauteur des sauts du $C M J$ sera supérieure à celle du $S J$. Les moyennes du pôle rugby testées par Cometti (2006) sont de 35,63 cm pour le $S J$ et de 44,67 cm pour le $C M J$ chez les avants, $36,03 \mathrm{~cm}(S J)$ et $48,70 \mathrm{~cm}(C M J)$ chez les arrières. Les moyennes chez les handballeurs sont de $38 \mathrm{~cm}(S J)$ et de 49,20 $\mathrm{cm}(C M J)$.

Ces différences entre le $S J$ et le $C M J$ peuvent être expliquées par un niveau élevé de pré-activité et d'activité musculaire lors de la phase excentrique durant le contremouvement, alors que dans le $S J$, cette activité se développe uniquement dans la phase de propulsion. Les muscles peuvent ainsi produire plus de force lors du CMJ (McBride et al., 2008) (Bobbert \& Casius, 2005). 
McCaulley et al. (2007) suggère que le cycle étirement-raccourcissement, qui inclue une phase intensive de freinage combinée à une forte activité simultanée musculaire, augmente le ratio entre le travail mécanique et la dépense énergétique. Cela peut être dû à la cinétique optimale de l'unité muscle-tendon et à l'utilisation de l'énergie élastique stockée.

Selon une étude de Gerodimos et al. (2008), l'amélioration de la performance maximale du $C M J$ par rapport au $S J$ s'explique par une augmentation du temps disponible pour développer la force, par la potentialisation des éléments contractiles et par la contribution des réflexes d'étirement et non pas par le stockage et l'utilisation de l'énergie élastique musculaire (Bobbert \& Casius, 2005) (Komi, 2000) (Van Ingen Schenau et al., 1997) (Komi \& Gollhofer, 1997) (Bobbert et al., 1996).

Les auteurs sont unanimes pour dire que la hauteur de saut est supérieure dans le $C M J$ par rapport au $S J$ mais les explications théoriques de cette différence varient.

\subsection{Objectif de notre revue}

L'objectif de notre revue systématique est d'évaluer les effets des différents types d'immersion (froide, contrastée) sur les DOMS, le facteur biochimique CK et la capacité fonctionnelle des membres inférieurs par rapport à divers groupes contrôles lors de la récupération musculaire post-effort de l'athlète.

\subsection{Question de recherche}

Quels sont les effets sur les DOMS, le facteur biochimique CK et la capacité fonctionnelle des membres inférieurs de l'immersion en eau (froide, contrastée) par rapport à divers groupes contrôles lors de la phase de récupération post-effort chez des sportifs pratiquant un sport acyclique? 


\section{Méthode}

\subsection{Design}

Nous avons réalisé une revue systématique de type quantitative et une méta-analyse en sélectionnant des essais randomisés contrôlés publiés dans la littérature scientifique entre les années 2000 et 2013.

\subsection{P.I.C.O.}

\subsubsection{Population}

La population des études incluses a ciblé des athlètes pratiquant un sport d'équipe de type acyclique.

\subsubsection{Intervention}

Nous avons intégré les articles utilisant l'immersion en phase de récupération (posteffort immédiate) des membres inférieurs (jusqu'à hauteur des crêtes iliaques / du nombril) en eau froide et/ou en eau contrastée.

\subsubsection{Comparaison}

Les études sélectionnées devaient comprendre au minimum un groupe d'immersion en eau froide et/ou contrastée et un groupe contrôle comprenant une intervention passive $(P A S)$ ou une autre modalité d'immersion.

\subsubsection{Outcomes}

Afin d'évaluer les effets de l'immersion, nous avons pris les deux indicateurs primaires suivants :

- un subjectif : les courbatures $(D O M S)$ pour le ressenti de l'athlète

- un marqueur biologique objectif : la créatine-kinase (CK).

Pour compléter notre travail, nous avons décidé de prendre un indicateur secondaire :

- la capacité fonctionnelle des membres inférieurs mesurée par le squat jump (SJ) ou le contre-mouvement jump (CMJ). 


\subsection{Critères d'inclusion}

Notre sélection s'est portée sur des sportifs amateurs et/ou professionnels afin d'augmenter l'échantillon. Nous avons opté pour une population masculine en se laissant le droit d'intégrer des études avec une population mixte car, suite à nos lectures, la majorité des publications analysait des sportifs de sexe masculin. Les sportifs devaient être âgés entre 16 et 40 ans car cela correspond, en général, aux extrêmes de la pratique d'un sport à un niveau actif en compétition.

\subsection{Critères d'exclusion}

Les études utilisant comme intervention l'immersion aquatique de niveau sternal ou corps entier, ou avec des protocoles de récupération associant l'immersion et une autre intervention (exemple : immersion et récupération active) ont été exclues. Les articles comprenant des sportifs pratiquant un sport cyclique ainsi que tous les sports se déroulant dans l'eau n'ont pas été pris en compte. Nous avons également éliminé les publications ne contenant aucun des indicateurs préalablement mentionnés. Les articles, rédigés dans des langues autres que le français, l'anglais, l'italien et l'allemand, n'ont pas été sélectionnés.

\subsection{Stratégie de recherche}

\subsubsection{Recherche électronique}

La recherche principale a été effectuée début octobre 2012 et a été complétée en mars 2013 afin d'intégrer les dernières publications scientifiques sur le sujet. Les bases de données suivantes ont été analysées : Pubmed, CINAHL, PEDro et Web of Science.

Nous avons combiné les opérateurs booléens $O R$ et $A N D$ avec les mots-clés suivants :

- « sports », « athlete»

- «water immersion », «water therapy », « hydrotherapy»

- «muscle soreness », «creatine-kinase », «jump », « recovery »

Nous avons adapté notre stratégie de recherche en fonction des caractéristiques propres à chaque base de données. 


\subsubsection{PubMed}

Nous avons utilisé des termes du thésaurus biomédical de référence (Medical Subject Headings, MeSH) et les trois catégories de mots-clés cités ci-dessus ainsi que leurs dérivés [cf. Annexe I] et avons répertorié 327 résultats.

\subsubsection{CINAHL}

Nous avons utilisé les trois catégories de mots-clés principaux cités ci-dessus ainsi que leurs dérivés [cf. Annexe I] et avons trouvé 24 résultats.

\subsubsection{PEDro}

Nous avons utilisé uniquement le mot-clé «immersion » pour cette base de données et avons recensé 54 résultats.

\subsubsection{Web of Science}

Nous avons combiné les termes «immersion », «sports », « recovery » grâce à l'opérateur booléen $A N D$ et avons obtenu 36 résultats.

\subsection{Sélection des articles}

Pour cette partie et à chaque étape de sélection, nous avons procédé de manière indépendante afin de comparer la concordance de nos résultats. En cas de divergences, une discussion s'est déroulée afin de trouver un accord quant à l'inclusion ou l'exclusion des articles problématiques.

\subsection{1 $1^{\text {er }}$ tri : sélection par titres, résumés et doublons}

La recherche sur les différentes bases de données a permis de réunir 441 articles. Afin d'effectuer le tri, ces derniers ont été listés dans un tableau sur Microsoft Office Excel ${ }^{\circledR}$. Nous avons retenu les articles mentionnant un sport et utilisant au moins un moyen de récupération par immersion aquatique. De plus, les indicateurs observés devant figurer dans les études étaient les suivants : les DOMS et/ou la CK et/ou la hauteur de saut vertical. Les études n'étant pas assez précises sur les outcomes observés ont été analysées dans leur version intégrale. 
Suite à cette étape, nous avons sélectionné 62 articles. Parmi ceux-ci, nous avons éliminé 31 doublons, portant à 31 le nombre de publications retenues. Cependant, durant ce processus, un doute s'est installé quant aux types de sport analysés (cyclique et/ou acyclique) car nous voulions être sûrs d'avoir un nombre suffisant d'article à examiner. Étant donné le nombre relativement élevé d'articles mentionnant un sport acyclique, nous avons décidé d'éliminer les 11 articles considérant les sports cycliques. Il nous restait ainsi 20 études.

\subsection{2 $2^{\text {ème }}$ tri : sélection par lecture de la version intégrale}

Nous avons rempli une grille permettant d'avoir une vue globale des différentes données PICO de l'étude ainsi que de son design. 15 articles ont été éliminés pour diverses raisons (cf. fluxogramme).

Afin d'obtenir un haut niveau de qualité de notre travail, nous nous sommes basés sur la pyramide de l'évidence en incluant uniquement des essais randomisés contrôlés car ils se situent au sommet de celle-ci et représentent un niveau de preuve élevé.

Un article a été écarté car il était impossible de l'obtenir malgré nos recherches et la prise de contact infructueuse avec l'auteur. Au final, nous avons retenu pour notre revue systématique 4 études. 


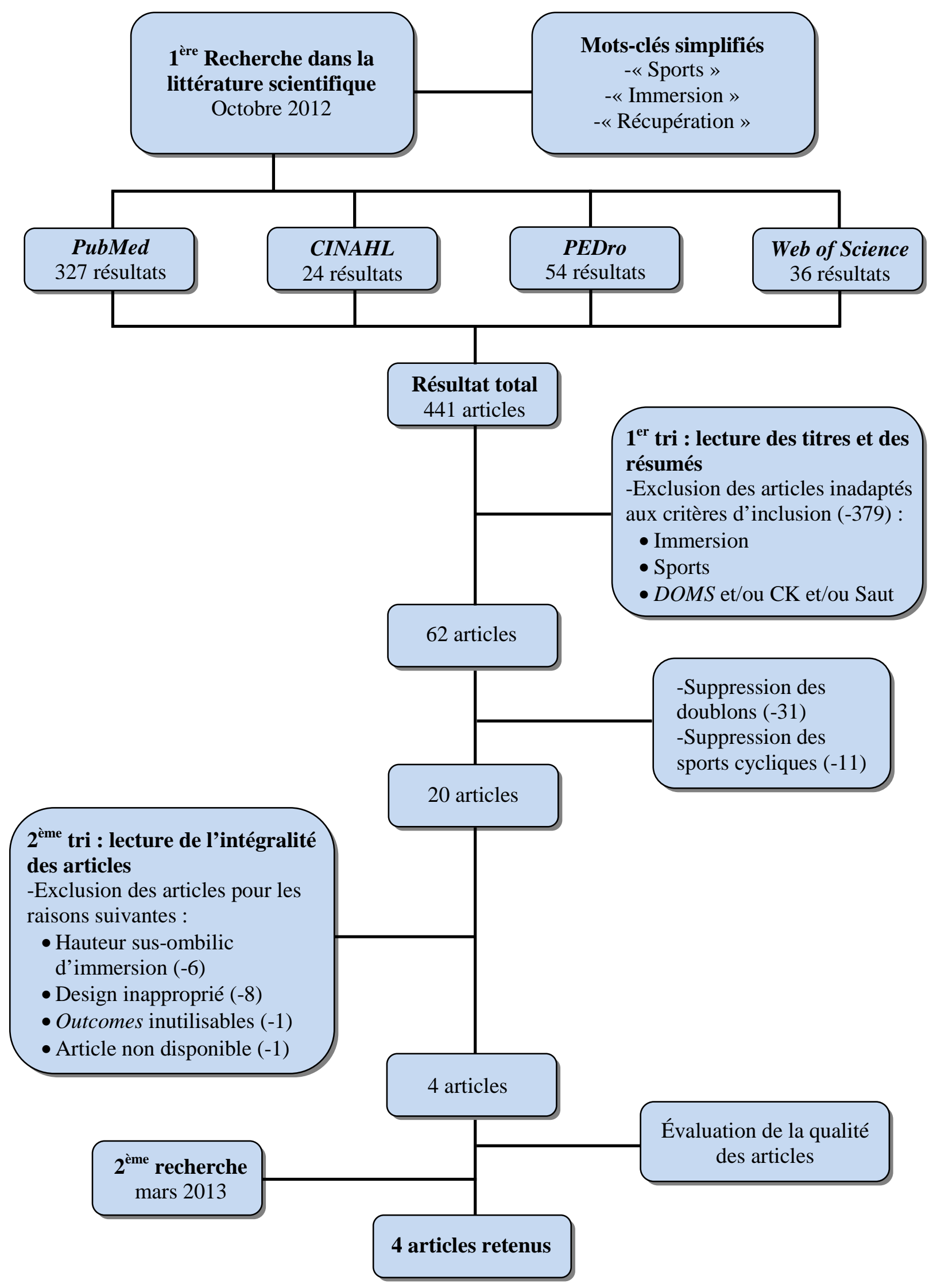

Figure 2 : fluxogramme de la sélection des articles 


\section{7 Évaluation de la qualité des articles}

Afin d'évaluer la validité interne et externe de nos quatre essais randomisés contrôlés, nous avons utilisé l'échelle PEDro (Physiotherapy Evidence Database) [cf. Annexe II] validé par Maher et al., (2003). Le score maximal de cette échelle est de 11 points. Nous avons attribué 1 point lorsque les critères stipulés étaient présents mais si un de ceux-ci n'était pas clairement établi ou carrément absent, aucun point n'a été accordé.

Cependant, nous avons décidé de baisser le total des points à 8. En effet, les trois critères «en aveugle» (sujets, thérapeutes et examinateurs) sont difficilement applicables dans le milieu physiothérapeutique et sportif. Étant donné la nature de l'intervention, il est impossible pour les sujets d'être «en aveugle». Cela se retrouve également au niveau de l'encadrement sportif et médical concernant le suivi de l'athlète. Il se peut que dans certaines études, les examinateurs soient «en aveugle » et qu'ils ne le soient pas dans d'autres. Au final, nous avons ainsi décidé d'inclure les études obtenant un score minimum de 5 sur 8 .

\subsubsection{Résultats de la qualité des études}

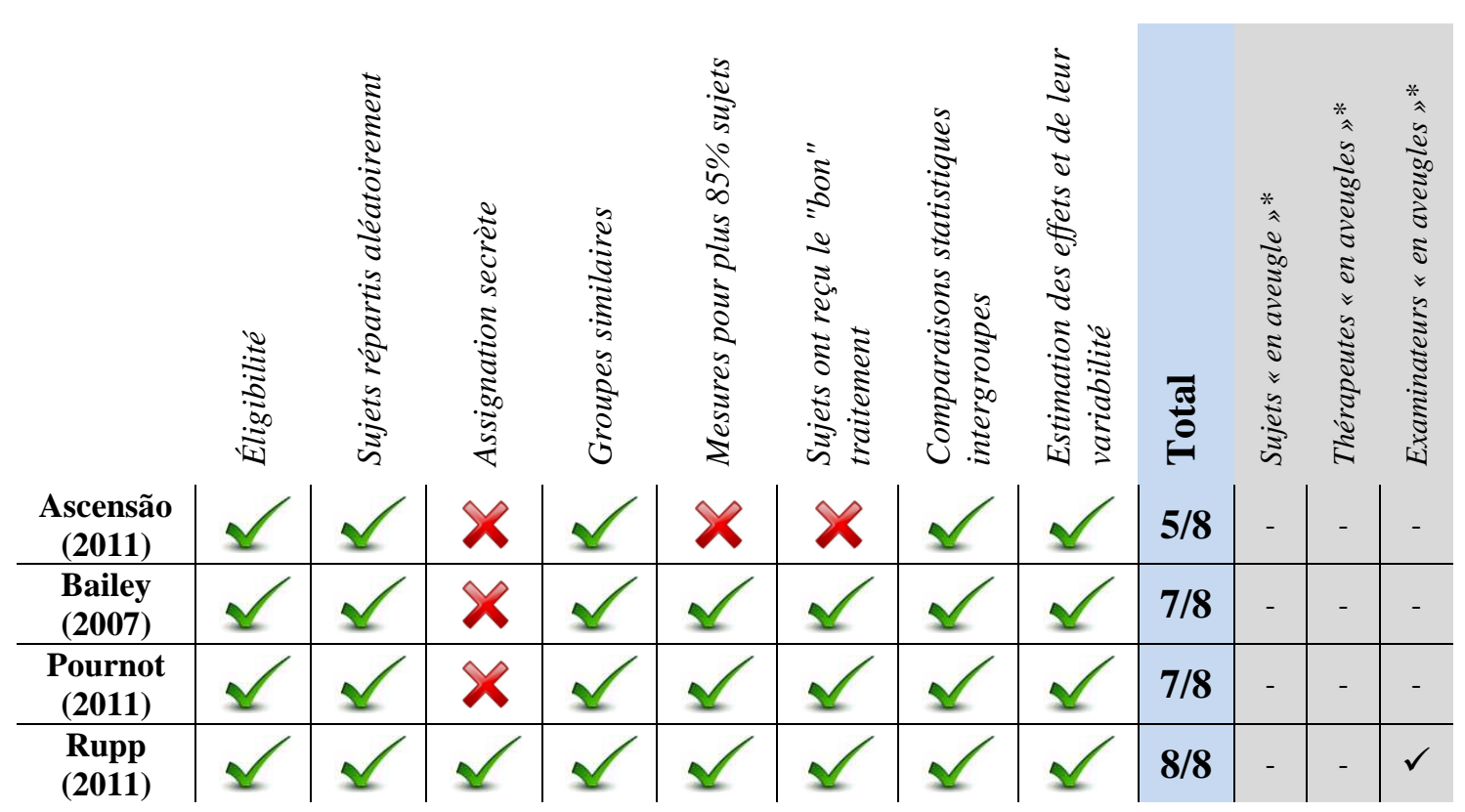

Tableau 1: évaluation de la qualité des articles grâce à l'échelle PEDro

* Ces 3 critères n'ont pas été pris en compte 


\section{8 Études incluses}

Après le tri des articles, nous avons retenu quatre essais randomisés contrôlés :

- Ascensão, A., Leite, M., Rebelo, A. N., Magalhäes, S., \& Magalhäes, J. (2011). Effects of cold water immersion on the recovery of physical performance and muscle damage following a one-off soccer match. Journal of Sports Sciences, $29(3), 217-225$.

- Bailey, D. M., Erith, S. J., Griffin, P. J., Dowson, A., Brewer, D. S., Gant, N., \& Williams, C. (2007). Influence of cold-water immersion on indices of muscle damage following prolonged intermittent shuttle running. Journal of sports sciences, 25(11), 1163-1170.

- Pournot, H., Bieuzen, F., Duffield, R., Lepretre, P.-M., Cozzolino, C., \& Hausswirth, C. (2011). Short term effects of various water immersions on recovery from exhaustive intermittent exercise. European Journal of Applied Physiology, 111(7), 1287-1295.

- Rupp, K. A., Selkow, N. M., Parente, W. R., Ingersoll, C. D., Weltman, A. L., \& Saliba, S. A. (2011). The Effect of Cold Water Immersion on 48 Hour Performance Testing in Collegiate Soccer Players. Exercise and Sport Injury Laboratory, University of Virginia. Journal of Strength and Conditioning Research / National Strength \& Conditioning Association. 


\subsubsection{Tableau 2 : données des études incluses}

\begin{tabular}{|c|c|c|c|c|c|c|c|c|}
\hline Auteurs & Titre & Design & Population & Sports & Activité physique & Interventions & Indicateurs & Temps \\
\hline $\begin{array}{l}\text { Ascensão et al. } \\
(2011)\end{array}$ & $\begin{array}{l}\text { Effects of cold water } \\
\text { immersion on the recovery } \\
\text { of physical performance } \\
\text { and muscle damage } \\
\text { following a one-off soccer } \\
\text { match }\end{array}$ & $\begin{array}{l}\text { Étude } \\
\text { randomisée } \\
\text { contrôlée }\end{array}$ & $\begin{array}{l}20 \widehat{\diamond} \\
18.1 \text { ans } \pm 1.8 \\
18.3 \text { ans } \pm 0.8\end{array}$ & Football & Match de football & $\begin{array}{l}-C W I: 10 \text { min. à } 10^{\circ} \mathrm{C} \\
-T W I: 10 \text { min. à } 35^{\circ} \mathrm{C}\end{array}$ & $\begin{array}{l}-D O M S \\
-\mathrm{CK} \\
-S J \\
-C M J\end{array}$ & $\begin{array}{l}\text {-Pré-activité } \\
-30 \text { min.* } \\
-24 \mathrm{H} \\
-48 \mathrm{H}\end{array}$ \\
\hline $\begin{array}{l}\text { Bailey et al. } \\
(2007)\end{array}$ & $\begin{array}{l}\text { Influence of cold-water } \\
\text { immersion on indices of } \\
\text { muscle damage following } \\
\text { prolonged intermittent } \\
\text { shuttle running }\end{array}$ & $\begin{array}{l}\text { Étude } \\
\text { randomisée } \\
\text { contrôlée }\end{array}$ & $\begin{array}{l}20 \AA \\
22.3 \text { ans } \pm 3.3\end{array}$ & $\begin{array}{l}\text { Football } \\
\text { Hockey } \\
\text { Rugby }\end{array}$ & $\begin{array}{l}\text { Ex. à intensités } \\
\text { variables pendant } \\
90 \text { min. à } 75 \% \text { de } \\
\text { la } \mathrm{VO}_{2} \text { max. }\end{array}$ & $\begin{array}{l}-C W I: 10 \text { min. à } 10^{\circ} \mathrm{C} \\
-P A S: \text { assis } 10 \text { min. à } \\
\text { température ambiante }\end{array}$ & $\begin{array}{l}-D O M S \\
-\mathrm{CK} \\
-S J\end{array}$ & $\begin{array}{l}\text {-Pré-activité } \\
-0 \mathrm{H} \\
-1 \mathrm{H} \\
-24 \mathrm{H} \\
-48 \mathrm{H} \\
-168 \mathrm{H}^{*}\end{array}$ \\
\hline $\begin{array}{l}\text { Pournot et al. } \\
\text { (2011) }\end{array}$ & $\begin{array}{l}\text { Short term effects of } \\
\text { various water immersions } \\
\text { on recovery from } \\
\text { exhaustive intermittent } \\
\text { exercise }\end{array}$ & $\begin{array}{l}\text { Étude } \\
\text { randomisée } \\
\text { contrôlée }\end{array}$ & $\begin{array}{l}41 \overbrace{}^{\lambda} \\
21.5 \text { ans } \pm 4.6\end{array}$ & $\begin{array}{l}\text { Football } \\
\text { Rugby } \\
\text { Volleyball }\end{array}$ & $\begin{array}{l}\text { Ex. intermittent } \\
\text { de } 2 \times 10 \text { min. } \\
\text { incluant des } C M J \\
\text { et du rameur }\end{array}$ & $\begin{array}{l}-C W I: 15 \text { min. à } 10^{\circ} \mathrm{C} \\
-C W T: \quad 15 \text { min. }(5 \mathrm{x} \\
\text { alterné : } 90 \text { sec. à } 10^{\circ} \mathrm{C}, 90 \\
\left.\text { sec. à } 42^{\circ} \mathrm{C}\right) \\
-T W I: 15 \text { min. à } 36^{\circ} \mathrm{C} \\
-P A S: \text { assis } 15 \text { min. à } \\
\text { température ambiante }\end{array}$ & $\begin{array}{l}-D O M S \\
-\mathrm{CK} \\
-C M J\end{array}$ & $\begin{array}{l}\text {-Pré-activité } \\
-0 \mathrm{H}^{*} \\
-1 \mathrm{H}^{*} \\
-24 \mathrm{H}\end{array}$ \\
\hline$\underset{(2011)}{\text { Rupp }}$ et al. & $\begin{array}{l}\text { The effect of cold water } \\
\text { immersion on } 48 \text {-hour } \\
\text { performance testing in } \\
\text { collegiate soccer players }\end{array}$ & $\begin{array}{l}\text { Étude } \\
\text { randomisée } \\
\text { contrôlée }\end{array}$ & $\begin{array}{l}13 \text { § et } 9 \text { ? } \\
19.8 \text { ans } \pm 1.1\end{array}$ & Football & Yo-yo Test & $\begin{array}{l}-C W I: 15 \text { min. à } 12^{\circ} \mathrm{C} \\
-P A S: \text { assis } 15 \text { min. à } \\
\text { température ambiante }\end{array}$ & $-C M J$ & $\begin{array}{l}\text {-Pré-activité } \\
-0 \mathrm{H} \\
-24 \mathrm{H} \\
-48 \mathrm{H}\end{array}$ \\
\hline
\end{tabular}

* les valeurs pour les temps mentionnés n'ont pas été prises pour tous les indicateurs.

Ex. correspond à exercice, $\mathrm{VO}_{2}$ max. à volume d'oxygène maximal, ${ }^{\circ} \mathrm{C}$ à température Celsius, sec. à seconde, min. à minute et $\mathrm{H}$ à heure. 


\subsection{Gestion des données}

\subsubsection{Données manquantes}

Malheureusement, dans les études, les résultats étaient majoritairement représentés sous forme de graphiques et il nous était impossible d'extraire des valeurs précises. De ce fait, une demande écrite a été envoyée à tous les auteurs afin d'obtenir les chiffres exactes des moyennes et des écart-types des différentes interventions.

Deux auteurs nous ont fourni les données susmentionnées et les deux restants nous ont donné les valeurs pour chaque participant, ce qui nous a permis de calculer la moyenne et la déviation standard à l'aide du programme Microsoft Excel ${ }^{\circledR}$.

Une autre difficulté est survenue lors de l'extraction des résultats de l'étude d'Ascensão et al. (2011) pour les DOMS, car cet indicateur a été évalué séparément pour les différents groupes musculaires des membres inférieurs. N'ayant pas reçu les données relatives à chaque participant, le calcul de la moyenne globale et de son écart-type n'était pas précis, mais tout de même envisageable. Cependant, nous avons remarqué que les DOMS au niveau des quadriceps étaient les plus élevées et avons jugé qu'elles étaient plus représentatives qu'une moyenne pondérée des différents groupes musculaires. Nous avons donc décidé de prendre uniquement les valeurs des DOMS des quadriceps.

\subsubsection{Extraction des données}

Nous avons réalisé des tableaux d'extraction des résultats comprenant les données suivantes [cf. Annexe III] :

- Nombre de participants

- Moyenne (Mean)

- Déviation standard (Standard Deviation, SD)

\subsection{Réalisation des forest plots}

Grâce à nos démarches, nous avons ensuite intégré toutes les données des études sélectionnées dans Review Manager $5^{\circledR}$ afin de les traiter et de les représenter sous 
forme de graphiques nommés forest plots. Chaque étude est symbolisée par un carré dont la taille varie proportionnellement au poids de l'étude. La ligne horizontale représente l'intervalle de confiance qui correspond à l'étendue des effets de l'étude. Le diamant démontre l'effet global de l'intervention par la sommation des études. Le positionnement des différents éléments permet de déduire en faveur de quelle intervention les résultats penchent. Un intervalle de confiance ou un diamant croisant l'axe vertical permet d'affirmer qu'il n'existe pas d'effet statistiquement significatif entre les interventions. Cependant, il est impossible de garantir que l'intervention ne présente pas d'effets cliniques (The Cochrane Collaboration, 2011).

Nous avons réalisé 15 forest plots, 5 pour chaque indicateur (DOMS, CK et hauteur de saut vertical) comparant la $C W I$, la $C W T$, la TWI et la PAS. 6 forest plots confrontant au moins deux études par rapport aux mêmes indicateurs et au même temps postintervention ont été analysés afin de réaliser une méta-analyse. Par souci de rigueur scientifique et pour représenter visuellement les résultats, nous avons effectué 9 autres forest plots [cf. Annexe IV] ne contenant qu'une seule étude.

Les forest plots sont analysés en tenant compte de l'hétérogénéité, de la valeur $\mathrm{P}$ et de l'intervalle de confiance.

\subsubsection{Hétérogénéité}

L'hétérogénéité $\left(\mathrm{I}^{2}\right)$ évalue la variabilité des effets des interventions entre les différentes études. Celle-ci est calculée automatiquement par le logiciel Review Manager $5^{\circledR}$. Pour son interprétation, nous nous sommes basés sur le chapitre 9.5.2 du manuel de Cochrane (The Cochrane Collaboration, 2011).

- $0-40 \%$ : pas de différence importante

- $30-60 \%$ : hétérogénéité modérée

- 50 - 90\% : hétérogénéité substantielle

- $75-100 \%$ : hétérogénéité considérable 


\subsubsection{Valeur $P$}

La valeur P représente la probabilité d'obtenir l'effet désiré par rapport à une hypothèse nulle $\left(\mathrm{H}_{0}\right)$ qui correspond à une «intervention sans effet clinique » ou à «aucune différence dans les effets des interventions entre les études ». Plus la valeur P est petite, plus elle indique que l'effet observé n'est pas dû au hasard. Une valeur $\mathrm{P}<0.05$ permet de rejeter l'hypothèse nulle et indique un effet statistiquement significatif. En revanche, une valeur P > 0.05 indique qu'il n'y a pas assez d'évidence pour prouver l'effet de l'intervention mais ne signifie pas que l'intervention n'a aucun effet. Afin d'éviter une mauvaise interprétation, la valeur $\mathrm{P}$ doit toujours être exprimée avec un intervalle de confiance à 95\% (The Cochrane Collaboration, 2011).

\subsubsection{Intervalle de confiance}

L'intervalle de confiance à $95 \%$ est un intervalle de valeurs qui a $95 \%$ de chance de contenir la vraie valeur du paramètre estimé. Si l'intervalle de confiance est relativement petit, la taille de l'effet est connue précisément. A contrario, lorsque l'intervalle est grand, l'incertitude de l'effet est grande et les effets sont peu connus (The Cochrane Collaboration, 2011). 


\section{Résultats}

\subsection{Analyse descriptive}

Notre revue systématique compile 4 études randomisées contrôlées publiées entre les années 2007 et 2011. Le nombre total est de 103 participants et se compose de 94 hommes et de 9 femmes. La moyenne d'âge des sujets est de 20.7 ans.

La répartition des individus dans les différentes interventions est la suivante :

- 45 pour la $C W I$ (4 études)

○ 20 à $10^{\circ} \mathrm{C}$ pendant $10 \mathrm{~min}$.

○ 13 à $10^{\circ} \mathrm{C}$ pendant $15 \mathrm{~min}$.

○ 12 à $12^{\circ} \mathrm{C}$ pendant $15 \mathrm{~min}$.

- 10 pour la $C W T$ (1 étude)

○ 10 alternativement $\left(5 \times 90 \mathrm{sec}\right.$.) à $10^{\circ} \mathrm{C}$ et $42^{\circ} \mathrm{C}$ pendant 15 min.

- 19 pour la TWI (2 études)

○ 10 à $35^{\circ} \mathrm{C}$ pendant $10 \mathrm{~min}$.

○ 9 à $36^{\circ} \mathrm{C}$ pendant $15 \mathrm{~min}$.

- 29 pour la $P A S$ (3 études)

○ 10 à température ambiante pendant 10 min.

○ 19 à température ambiante pendant $15 \mathrm{~min}$.

Chaque immersion a été effectuée à hauteur des crêtes iliaques ou du nombril.

En ce qui concerne les indicateurs, le nombre de sujets évalués est de :

- 81 pour les DOMS (3 études) dont 61 mesurés sur une EVA comprise entre 0 et 10 et 20 mesurés sur une EVA comprise entre 1 et 10.

- 79 pour la CK (3 études) mesurés en U/L.

- 102 pour le saut dont 20 pour le $S J$ (1 étude) et 82 pour le $C M J$ (3 études) mesurés en $\mathrm{cm}$.

Toutes ces données ont été analysées de manières inférentielle et narrative afin d'évaluer les effets de l'immersion en eau froide ou contrastée par rapport à un groupe contrôle. 


\subsection{Analyse inférentielle}

\subsubsection{Douleurs musculaires d'apparition retardée à $24 \mathrm{H}$}

Afin d'analyser les données statistiques de l'immersion pour les DOMS, nous avons compilé les résultats des études de Bailey et al. (2007) et de Pournot et al. (2011) pour comparer la $C W I$ et la $P A S$ ainsi que celles d'Ascensão et al. (2011) et de Pournot et al. (2011) pour confronter la CWI et la TWI.

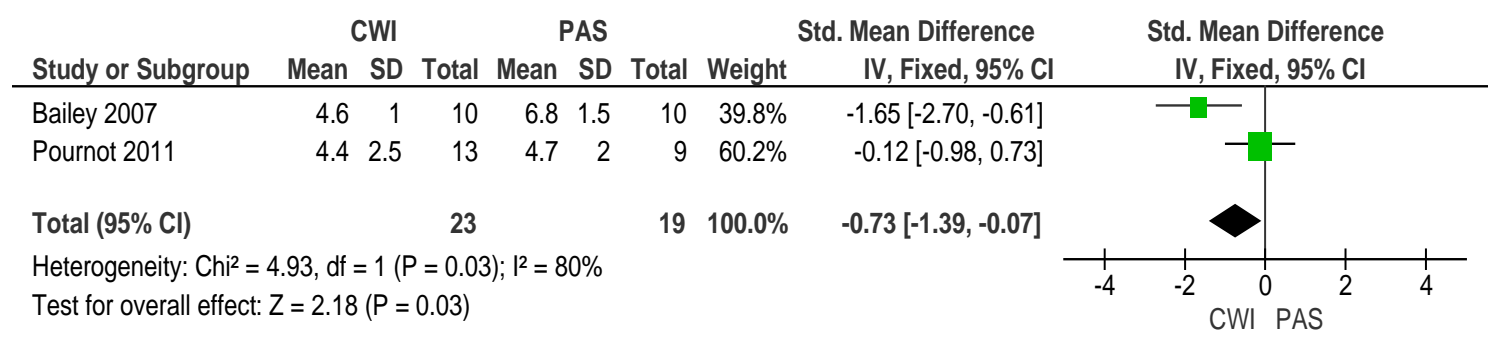

Tableau 3 : comparaison entre la $C W I$ et la $P A S$ pour les $D O M S$ à $24 \mathrm{H}$

Deux études comprenant 23 sujets dans le groupe $C W I$ et 19 dans le groupe $P A S$ ont été collectées et examinées. Cette analyse des données montre une différence statistiquement significative $(\mathrm{P}=0.03)$ en faveur de la $C W I$ s'élevant à -0.73 point [95\% CI -1.39, -0.07]. L'hétérogénéité $\left(\mathrm{I}^{2}\right)$ est à hauteur de $80 \%$ (hétérogénéité considérable).

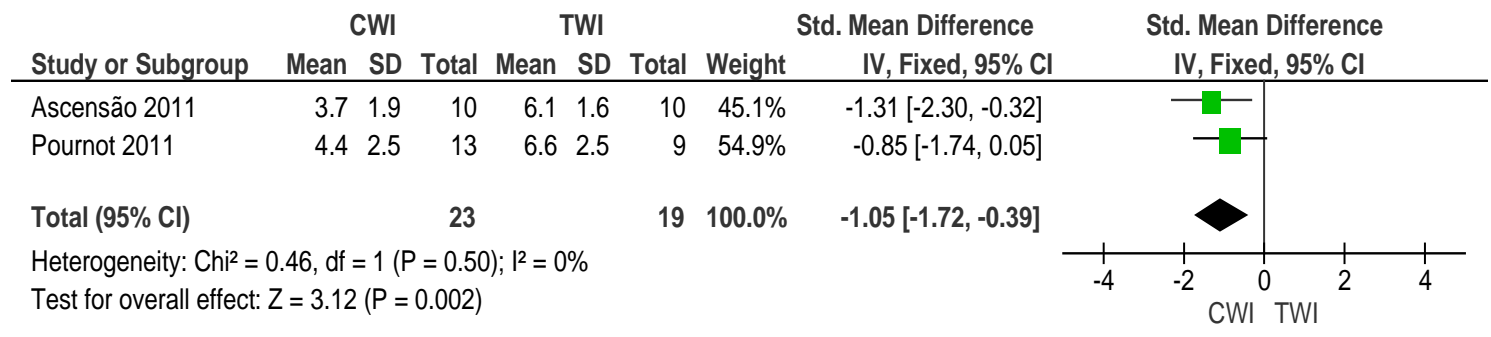

Tableau 4 : comparaison entre la $C W I$ et la $T W I$ pour les DOMS à $24 \mathrm{H}$

Deux études comprenant 23 sujets dans le groupe $C W I$ et 19 dans le groupe TWI ont été collectées et examinées. Cette analyse des données montre une différence statistiquement significative $(\mathrm{P}=0.002)$ en faveur de la $C W I$ s'élevant à -1.05 point [95\% CI -1.72, -0.39]. L'hétérogénéité $\left(\mathrm{I}^{2}\right)$ est à hauteur de $0 \%$ (pas de différence importante). 


\subsubsection{Créatine-kinase à $24 \mathrm{H}$}

Afin d'analyser les données statistiques de l'immersion pour la CK, nous avons compilé les résultats des études de Bailey et al. (2007) et de Pournot et al. (2011) pour comparer la $C W I$ et la PAS ainsi que celles d'Ascensão et al. (2011) et de Pournot et al. (2011) pour confronter la $C W I$ et la $T W I$.

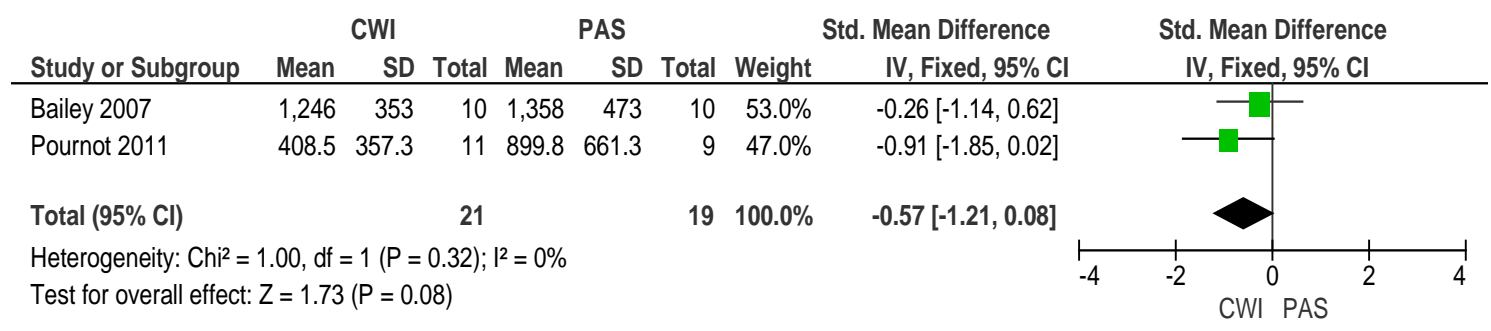

Tableau 5 : comparaison entre la $C W I$ et la $P A S$ pour la $C K$ à $24 \mathrm{H}$

Deux études comprenant 21 sujets dans le groupe $C W I$ et 19 dans le groupe $P A S$ ont été collectées et examinées. Cette analyse des données montre une différence statistiquement non-significative $(\mathrm{P}=0.08)$ en faveur de la $C W I$ s'élevant à -0.57 point [95\% CI -1.21, 0.08]. L'hétérogénéité $\left(\mathrm{I}^{2}\right)$ est à hauteur de $0 \%$ (pas de différence importante).

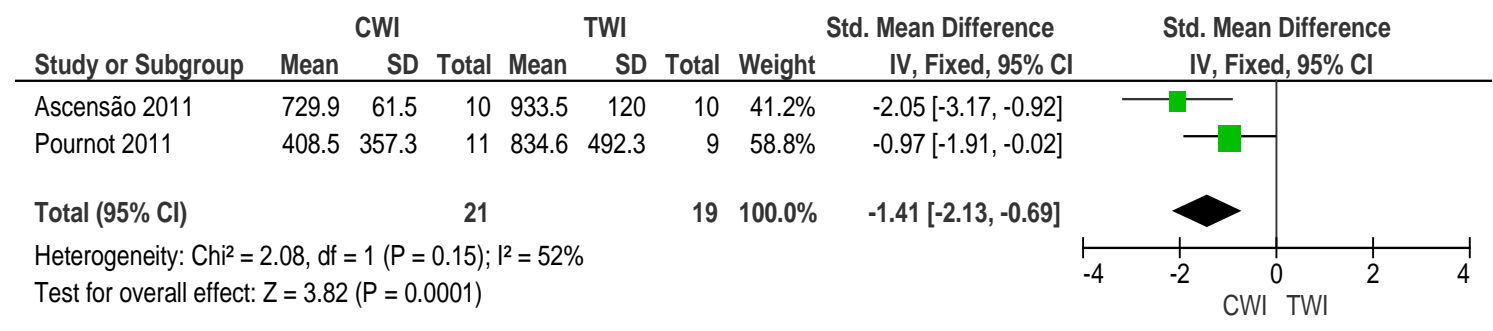

Tableau 6 : comparaison entre la $C W I$ et la $T W I$ pour la CK à $24 \mathrm{H}$

Deux études comprenant 21 sujets dans le groupe $C W I$ et 19 dans le groupe TWI ont été collectées et examinées. Cette analyse des données montre une différence statistiquement significative $(\mathrm{P}=0.0001)$ en faveur de la $C W I$ s'élevant à -1.41 point [95\% CI -2.13, -0.69]. L'hétérogénéité $\left(\mathrm{I}^{2}\right)$ est à hauteur de 52\% (hétérogénéité substantielle). 


\subsubsection{Capacité fonctionnelle des membres inférieurs à $24 \mathrm{H}$}

Afin d'analyser les données statistiques de l'immersion pour le $S J$ et le $C M J$, nous avons compilé les résultats des études de Pournot et al. (2011) et de Rupp et al. (2011) pour comparer la $C W I$ et la $P A S$ ainsi que celles d'Ascensão et al. (2011) et de Pournot et al. (2011) pour confronter la $C W I$ et la $T W I$.

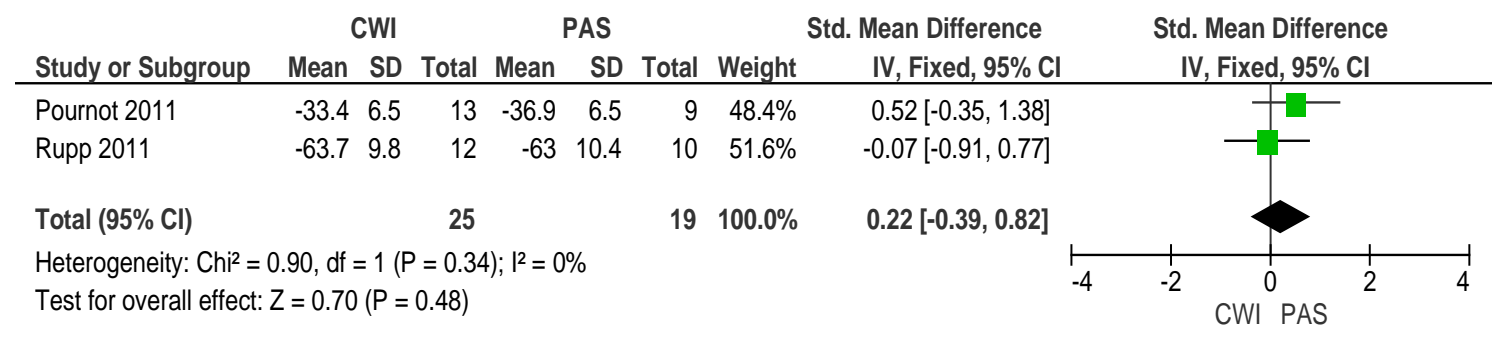

Tableau 7 : comparaison entre la $C W I$ et la $P A S$ pour la hauteur de saut à $24 \mathrm{H}$

Deux études comprenant 25 sujets dans le groupe $C W I$ et 19 dans le groupe PAS ont été collectées et examinées. Cette analyse des données montre une différence statistiquement non-significative $(\mathrm{P}=0.48)$ en faveur de la $P A S$ s'élevant à 0.22 point [95\% CI -0.39, 0.82]. L’hétérogénéité $\left(\mathrm{I}^{2}\right)$ est à hauteur de $0 \%$ (pas de différence importante).

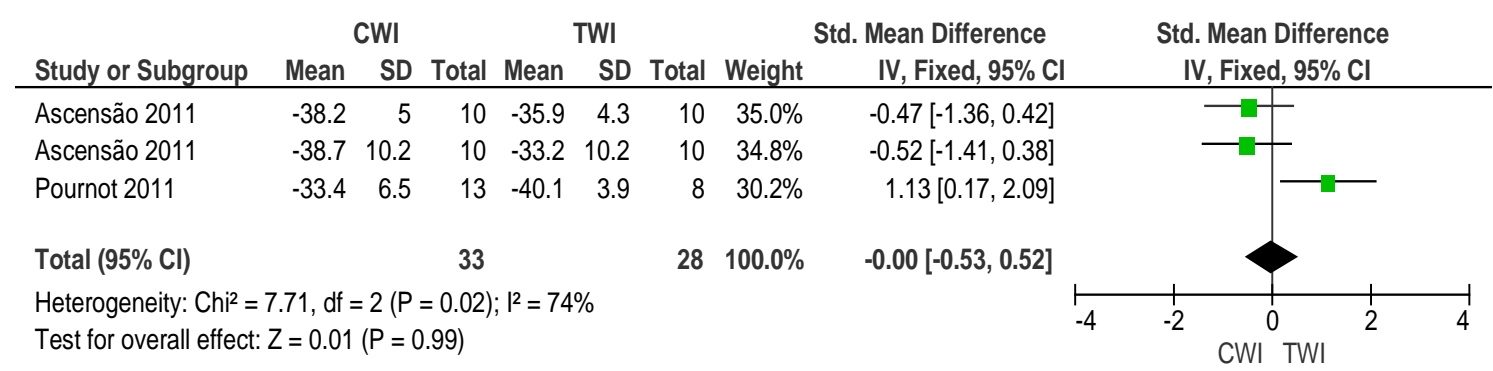

Tableau 8 : comparaison entre la $C W I$ et la $T W I$ pour la hauteur de saut à $24 \mathrm{H}$

Trois études comprenant 33 sujets dans le groupe $C W I$ et 28 dans le groupe TWI ont été collectées et examinées. Cette analyse des données ne montre aucune différence (statistiquement non-significative, $\mathrm{P}=0.99$ ) entre les deux groupes s'élevant à -0.00 point $[95 \%$ CI $-0.53,0.52]$. L'hétérogénéité $\left(\mathrm{I}^{2}\right)$ est à hauteur de $74 \%$ (hétérogénéité substantielle). 


\subsubsection{Récapitulatif des résultats des forest plots}

Le tableau ci-dessous synthétise les différents résultats obtenus par l'analyse des forest plots et permet une vue d'ensemble de l'effectivité des diverses interventions.

Tableau 9 : synthèse des résultats obtenus

\begin{tabular}{|l|c|c|}
\hline & $C W I$ vs $P A S$ & $C W I$ vs TWI \\
\hline DOMS à 24H & $\swarrow$ & \\
\cline { 2 - 3 } CK à 24H & $\swarrow$ & \\
\cline { 2 - 3 } Saut à 24H & $\searrow$ & \\
\hline
\end{tabular}

En faveur de la $C W I$ (statistiquement significatif)

1 En faveur de la $C W I$ (statistiquement non-significatif)
En faveur de la $P A S$ (statistiquement non-significatif) Aucune différence entre $C W I$ et $T W I$

Pour les $D O M S$ et la $\mathrm{CK}$ à $24 \mathrm{H}$, quatre comparaisons sont en faveur de la $C W I$ par rapport à la $P A S$ et la $T W I$, dont trois sont statistiquement significatives $(\mathrm{P}<0.05)$. Pour la hauteur de saut à $24 \mathrm{H}$, une comparaison pencherait pour la $P A S$ (différence statistiquement non-significatif, $\mathrm{P}>0.05$ ) par rapport à la $C W I$ et une autre comparaison ne montre aucune différence entre la $C W I$ et la $T W I$.

\subsection{Analyse narrative}

\subsection{1 Étude d'Ascensão et al. (2011)}

Cette étude randomisée contrôlée a comparé les effets de la CWI par rapport à la $T W I$ sur les dysfonctions et les dommages musculaires suite à un match de football de 90 minutes chez 20 jeunes footballeurs.

Le premier groupe $(\mathrm{N}=10,18.1$ ans \pm 1.4$)$ a reçu la $C W I\left(10^{\circ} \mathrm{C}, 10 \mathrm{~min}\right.$.) pendant que l'autre $(\mathrm{N}=10,18.3$ ans \pm 0.8$)$ a bénéficié de la $T W I\left(35^{\circ} \mathrm{C}, 10 \mathrm{~min}\right.$. $)$. Les auteurs ont analysé les DOMS de plusieurs groupes musculaires (quadriceps, ischio-jambiers, triceps suraux, adducteurs) et les taux sanguins de CK, de myoglobine et de protéine Créactive. D'autres données fonctionnelles ont été prises en compte comme le $S J$, le $C M J$, l'habileté au sprint de 20 mètres et la force maximale isométrique de contraction 
volontaire. Les mesures ont été collectées avant le match, à 30 min. post-intervention, à $24 \mathrm{H}$ et à $48 \mathrm{H}$.

L'étude a démontré une différence statistiquement significative $(\mathrm{P}<0.05)$ en faveur du groupe $C W I$ par rapport au groupe $T W I$ pour les DOMS (quadriceps) à $24 \mathrm{H}$ mais aucune à 30 min. et à $48 \mathrm{H}$. Pour la $\mathrm{CK}$, il y a une différence statistiquement significative $(\mathrm{P}<$ $0.05)$ à $24 \mathrm{H}$ et à $48 \mathrm{H}$ en faveur du groupe $C W I$. En ce qui concerne le $S J$ et le $C M J$, il n'y a pas de différence statistiquement significative $(\mathrm{P}>0.05)$ entre les deux groupes.

\subsection{2 Étude de Bailey et al. (2007)}

Cette étude randomisée contrôlée a évalué les effets de la CWI sur les indices de dommages musculaires après des exercices intermittents prolongés (90 minutes) chez 20 hommes pratiquant différents sports d'équipes (football, hockey et rugby).

Ils ont été divisés en un groupe expérimental $(\mathrm{N}=10,23.6$ ans \pm 4.1$)$ utilisant la $C W I$ $\left(10^{\circ} \mathrm{C}, 10 \mathrm{~min}.\right)$ et en un groupe contrôle $(P A S)(\mathrm{N}=10,21.7$ ans \pm 2.0$)$ restant assis 10 minutes. Les indicateurs mesurés étaient les $D O M S$, la $\mathrm{CK}$, le $S J$, la contraction maximale isométrique volontaire et la vitesse de sprint. Les données ont été récoltées avant l'exercice, immédiatement après, à $1 \mathrm{H}$, à $24 \mathrm{H}$, à $48 \mathrm{H}$ et à $168 \mathrm{H}$.

Les résultats de l'étude ont mis en évidence une différence statistiquement significative $(\mathrm{P}<0.05)$ pour les $D O M S$ en faveur du groupe $C W I$ à $1 \mathrm{H}, 24 \mathrm{H}$ et $48 \mathrm{H}$. Pour la $\mathrm{CK}$ et le $S J$, aucune différence statistiquement significative n'a été retrouvée $(\mathrm{P}>0.05)$ entre les deux groupes.

\subsection{3 Étude de Pournot et al. (2011)}

Cette étude randomisée contrôlée a analysé les effets à court terme de différents types d'immersion aquatique suite à des exercices intermittents (2x 10 min.) chez 41 hommes entrainés (21.5 ans \pm 4.6 ) (football, rugby ou volleyball).

Les chercheurs ont formé un groupe $C W I\left(\mathrm{~N}=13,10^{\circ} \mathrm{C}, 15 \mathrm{~min}\right.$.), un groupe $C W T$ $\left(\mathrm{N}=10,5 \mathrm{x}\right.$ alternativement $90 \mathrm{sec}$. à $10^{\circ} \mathrm{C}$ et $90 \mathrm{sec}$. à $42^{\circ} \mathrm{C}, 15 \mathrm{~min}$.), un groupe $T W I$ $\left(\mathrm{N}=9,36^{\circ} \mathrm{C}, 15 \mathrm{~min}\right.$.) et un groupe contrôle $P A S(\mathrm{~N}=9)$ restant assis 15 minutes. Les indicateurs observés étaient les $D O M S$, la $\mathrm{CK}$, le $C M J$, la contraction maximale 
isométrique volontaire et la puissance. Les données ont été mesurées avant l'exercice, immédiatement après, à $1 \mathrm{H}$ et à $24 \mathrm{H}$.

Les résultats n'ont pas donné de différence statistiquement significative $(\mathrm{P}>0.05)$ entre les quatre groupes d'intervention pour les $D O M S$, la $C M J$ et le CK. Cependant, pour ce dernier, il n'y a pas eu de différence statistiquement significative $(P>0.05)$ dans l'augmentation des valeurs de base et des valeurs à $24 \mathrm{H}$ pour la $C W I$, contrairement à la $P A S, T W I$ et $C W T$. En ce qui concerne le $C M J$ et dans chaque intervention, il n'y a pas eu de différence statistiquement significative avec les valeurs de base $(\mathrm{P}>0.05)$ et les valeurs à $24 \mathrm{H}$. Cela a également été le cas pour la $C W I$ à $1 \mathrm{H}$ post-exercice.

Une différence entre les résultats de l'étude de Pournot et al. (2011) et ceux de nos forest plots a été constatée [cf. Annexe IV]. Cependant, nous avons tenu compte des résultats de l'article car nous ne pouvions pas expliquer l'origine de ces différences.

\subsection{4 Étude de Rupp et al. (2011)}

Cette étude randomisée contrôlée a examiné l'effet de l'immersion en eau froide comme une modalité de récupération suite à un exercice intermittent (yo-yo test) induisant une fatigue musculaire chez 22 jeunes footballeurs (13 ðै et 9 q, 19.8 ans \pm 1.1 ).

Ils ont été séparés en un groupe expérimental bénéficiant de la $C W I\left(\mathrm{~N}=12,12^{\circ} \mathrm{C}, 15\right.$ min.) et un groupe contrôle $P A S(\mathrm{~N}=10)$ restant assis 15 minutes. Une deuxième session d'intervention a été effectuée 24 heures post-immersion. Les indicateurs observés étaient le $C M J$, la perception de la fatigue des membres inférieurs, la perception de l'effort et le yo-yo test. Ils ont été mesurés avant l'intervention, à $24 \mathrm{H}$ et à $48 \mathrm{H}$.

Les résultats du $C M J$ n'ont montré aucune différence statistiquement significative (P > 0.05 ) entre les deux interventions à $24 \mathrm{H}$ et à $48 \mathrm{H}$. Cependant, il n'y a pas eu de diminution statistiquement significative des valeurs du $C M J$ entre les valeurs de base et celles à $24 \mathrm{H}$ pour la $C W I$, contrairement au groupe $P A S$. 


\section{Discussion}

L'objectif de notre revue était d'évaluer les effets de l'immersion en eau froide ou contrastée sur les DOMS, le facteur biochimique CK et la capacité fonctionnelle des membres inférieurs lors de la récupération musculaire post-effort de l'athlète. Nos résultats sont favorables à l'utilisation de la $C W I$ comme moyen de récupération dans les sports acycliques. Plus précisément, ils démontrent un effet positif de cette technique sur la réduction des DOMS et du taux de $\mathrm{CK}$ à $24 \mathrm{H}$.

En raison de nos critères de sélection et des études explorées, nous n'avons pas comparé de manière statistique les effets de la $C W T$ par rapport aux autres interventions. Cependant, nous allons tout de même les aborder en utilisant l'analyse narrative de l'étude de Pournot et al. (2011) et en se référant à la littérature scientifique.

Les résultats de nos forest plots sont applicables à une hauteur d'immersion au niveau des crêtes iliaques. Pour la $C W I$, les températures de l'eau sont comprises entre $10^{\circ} \mathrm{C}$ et $12^{\circ} \mathrm{C}$ et les durées varient de 10 à 15 minutes. En ce qui concerne la $C W T$, les résultats proviennent de l'étude de Pournot et al. (2011) utilisant le protocole d'immersion suivant : cinq cycles alternés à $10^{\circ} \mathrm{C}$ pendant 90 secondes et à $42^{\circ} \mathrm{C}$ pendant 90 secondes pour une durée totale de 15 minutes.

\subsection{Douleurs musculaires d'apparition retardée}

Notre analyse des études sélectionnées démontre l'utilité de l'immersion en eau froide afin de réduire la valeur des DOMS à $24 \mathrm{H}$ post-effort chez des sportifs acycliques. En effet, les résultats de la $C W I$ montrent une diminution statistiquement significative des $D O M S$ à $24 \mathrm{H}$ par rapport à d'autres modalités de récupération (PAS et $T W I)$.

Dans la littérature, nous retrouvons trois études (Delextrat et al., 2012) (Pointon et al., 2011) (Ingram et al., 2009) similaires en termes de modalités de récupération et de sports pratiqués. Les résultats de ces études indiquent une différence statistiquement significative en faveur de la $C W I$ par rapport à la $P A S$ à $24 \mathrm{H}$ pour les courbatures des membres inférieurs. Cependant, nous retrouvons également un article de Pointon \& 
Duffield (2012) qui ne montre pas de différence statistiquement significative entre la $C W I$ et la $P A S$ à $24 \mathrm{H}$ pour les $D O M S$ au niveau des membres inférieurs sur des athlètes acycliques.

Nous remarquons que ces résultats d'articles à buts similaires tendent à opter pour l'utilisation de la $C W I$ afin de diminuer les DOMS à $24 \mathrm{H}$ post-effort. Toutefois, le niveau de preuve scientifique est moins élevé car les études citées précédemment sont de type cross-over.

Concernant la CWT, l'étude de Pournot et al. (2011) ne démontre pas de différence significative en comparaison des trois autres modalités de récupération (CWI, TWI et $P A S$ ) pour les courbatures à $24 \mathrm{H}$.

Dans la littérature, une étude d'Ingram et al. (2009) compare sur onze athlètes de sports collectifs les modalités de récupération suivantes : la $C W I$, la $C W T$ et la $P A S$. Les résultats indiquent une différence statistiquement significative en faveur de la $C W T$ par rapport à la $P A S$ à $24 \mathrm{H}$ pour les $D O M S$ au niveau des membres inférieurs. Par contre, l'utilisation de la $C W I$ montre une différence statistiquement significative dans la réduction de cet indicateur à $24 \mathrm{H}$ par rapport à la $C W T$.

Notre méta-analyse ainsi que le résultat de cette étude ne permettent pas de justifier l'utilisation de la $C W T$ à $24 \mathrm{H}$ post-effort pour la réduction des $D O M S$ des membres inférieurs.

En conclusion, nous proposons d'utiliser la $C W I$ comme technique de récupération pour réduire les $D O M S$ à $24 \mathrm{H}$ suite à la pratique d'un sport acyclique.

\subsection{Créatine-kinase}

Les résultats de notre méta-analyse montrent un certain intérêt de l'utilisation de la $C W I$ pour obtenir un taux plus bas de CK à $24 \mathrm{H}$ post-effort chez des sportifs acycliques. En effet, la $C W I$ possède une différence statistiquement significative par rapport à la $T W I$ et une différence statistiquement non-significative en faveur de la $C W I$ par rapport à la $P A S$. De plus, dans l'étude de Pournot et al., (2011), la CWI est la seule modalité de 
récupération qui ne possède pas de différence significative entre les valeurs de base et les valeurs à $24 \mathrm{H}$, contrairement aux trois autres $(C W T, T W I, P A S)$. Cela sous-entend que l'augmentation du niveau de CK est limitée suite à la $C W I$.

Dans la littérature, nous retrouvons deux études (Pointon \& Duffield, 2012) (Pointon et al., 2011) comparant la CWI et la PAS et une étude (Ingram et al., 2009) qui confronte la $C W I$, la $C W T$ et la $P A S$ chez des athlètes de sports collectifs. Les résultats de ces trois articles sont unanimes pour dire qu'il n'y a pas de différence statistiquement significative entre ces trois modalités de récupération à $24 \mathrm{H}$ pour la $\mathrm{CK}$. Une étude randomisée contrôlée de De Nardi et al. (2011) sur dix-huit jeunes footballeurs compare la $C W I$, la $C W T$ et la $P A S$. Les résultats indiquent un taux d'augmentation plus bas de $\mathrm{CK}$ de manière statistiquement significative en faveur de la $C W I$ par rapport à la $P A S$ à $96 \mathrm{H}$ post-effort.

Ces observations ne permettent pas de justifier l'utilisation de la $C W I$ pour limiter l'augmentation du taux de $\mathrm{CK}$ à $24 \mathrm{H}$ post-effort car les résultats ci-dessus ne sont pas clairement en faveur d'une des modalités. Cependant, nous remarquons tout de même un effet clinique de la $C W I$ sur cet indicateur biochimique.

Concernant la $C W T$, l'étude de Pournot et al. (2011) ne démontre pas de différence significative en comparaison des trois autres modalités de récupération (CWI, TWI, $P A S$ ) pour la CK à $24 \mathrm{H}$.

Dans la littérature, l'étude d'Ingram et al., (2009) n'indique pas de différence statistiquement significative entre la $C W T$ et la $P A S$ pour la $\mathrm{CK}$ à $24 \mathrm{H}$ post-effort. Cependant, dans un autre article, Gill et al. (2006) compare la CWT, la PAS et deux autres modalités de récupération sur vingt-trois rugbymen. Les résultats révèlent une différence statistiquement significative en faveur de la $C W T$ pour cet indicateur par rapport à la $P A S$ à $36 \mathrm{H}$ et à $84 \mathrm{H}$ post-effort. De plus, l'étude de De Nardi et al. (2011) montre un résultat statistiquement significatif en faveur de la $C W T$ par rapport à la $P A S$ pour la $\mathrm{CK}$ à $96 \mathrm{H}$ mais pas de différence entre la $C W I$ et la $C W T$.

Les résultats obtenus ne nous permettent pas de conseiller l'utilisation de la $C W T$ pour atténuer l'augmentation du taux de CK à $24 \mathrm{H}$ post-effort. En revanche, certaines études 
montrent un effet sur ce marqueur biochimique à des temps post-efforts plus éloignés par rapport à la $P A S$.

En résumé, l'utilisation de la $C W I$ est cliniquement bénéfique afin de limiter l'augmentation du taux de $\mathrm{CK}$ à $24 \mathrm{H}$ post-effort. Par contre, nos résultats ne sont pas en faveur de la $C W T$, même si divers auteurs mentionnent certains effets pour cet indicateur.

\subsection{Capacité fonctionnelle des membres inférieurs}

Les résultats de notre travail ne montrent aucun intérêt à utiliser la $C W I$ pour la hauteur de saut vertical à $24 \mathrm{H}$ post-effort chez des sportifs acycliques. En effet, la $C W I$ ne présente aucune différence par rapport à la $T W I$ et les résultats de la comparaison entre la $C W I$ et la $P A S$ établissent une différence statistiquement non-significative en faveur de la PAS. De plus, l'étude de Bailey et al. (2007) démontre qu'il n'y a aucune différence entre l'utilisation de la $C W I$ et de la $P A S$ et appuie ainsi nos résultats.

Dans la littérature, nous retrouvons une étude de type cross-over de Delextrat et al. (2012) qui compare la $C W I$, la $P A S$ et le massage chez huit hommes et huit femmes pratiquant le basketball. Les résultats indiquent une différence statistiquement significative en faveur de la $C W I$ par rapport à la $P A S$ à $24 \mathrm{H}$ pour le $C M J$. Une étude de De Nardi et al. (2011), ne montre pas de différence statistiquement significative entre la $C W I$, la $C W T$ et la $P A S$ à $24 \mathrm{H}$ pour le $C M J$. Toutefois, les résultats à $72 \mathrm{H}$ exposent une tendance en faveur de la $C W I$ et de la $C W T$ par rapport à la $P A S$ pour le $C M J$ mais qui n'est pas statistiquement significatif.

Concernant la $C W T$, l'étude de Pournot et al. (2011) ne présente pas de différence significative en comparaison des trois autres modalités de récupération ( $C W I, T W I$, $P A S$ ) pour la hauteur de saut vertical à $24 \mathrm{H}$. La recherche d'articles similaires à nos critères de sélection s'est avérée infructueuse et ne nous permet pas de confronter nos résultats à la littérature. 
Notre méta-analyse ainsi que la littérature scientifique analysée démontrent que la $C W I$ et la $C W T$ ne permettent pas d'atténuer la perte des capacités fonctionnelles des membres inférieurs à $24 \mathrm{H}$ post-effort et ne représentent aucun intérêt.

\subsection{Autres paramètres observés dans la littérature}

Lors de l'exploration de la littérature, en analysant les interventions menées, nous avons constaté que la hauteur d'immersion s'effectue aussi jusqu'au niveau du sternum ou des épaules. En parcourant les études possédant ce critère, la majorité des résultats s'avéraient être similaires à notre discussion.

Pour les DOMS, quatre études (Elias et al. ${ }^{1}, 2012$ ) (Elias, et al. ${ }^{2}, 2012$ ) (Rowsell et al., 2011) (Rowsell et al., 2009) mentionnent l'efficacité de la CWI par rapport à d'autres modalités de récupération $(C W T, T W I$ et $P A S)$ à des temps post-efforts variables. Toutefois, une étude (Vaile et al., 2008) indique une réduction statistiquement significative des DOMS suite à la $C W T$ en comparaison de la $P A S$ mais pas pour la CWI.

Une publication de Vaile et al. (2008) démontre une différence statistiquement significative en faveur de la $C W I$ par rapport à la $P A S$ pour le taux de $\mathrm{CK}$ post-effort à 24H. Toutefois, Rowsell et al. (2009), dans son étude, ne retrouve pas de différence entre l'utilisation de la $C W I$ et de la $T W I$.

Pour la capacité fonctionnelle des membres inférieurs, des résultats contradictoires sont décrits. Effectivement, deux études (Rowsell et al., 2009) (Kinugasa \& Kilding, 2009) ne révèlent pas de différence entre la $C W I$ et diverses modalités (CWT, TWI et PAS) tandis que d'autres articles (Elias et al. ${ }^{1}, 2012$ ) (Elias et al. ${ }^{2}, 2012$ ) prônent l'utilisation de la $C W I$ par rapport à la $P A S$ et à la $C W T$. Cette dernière démontre tout de même un effet marqué par rapport à la $P A S$ (Elias et al. ${ }^{2}, 2012$ ). À l'inverse, une étude de (Vaile et al., 2008) préconise l'utilisation de la $C W T$ par rapport à la $C W I$ et la PAS en testant le $S J$ de manière lestée.

D'autres indicateurs sont également analysés par certains auteurs pour évaluer les effets de l'immersion comme la force maximale isométrique des membres inférieurs, les 
sprints, la fatigue, ou encore d'autres marqueurs biologiques (lactates, leukocytes, protéine $\mathrm{C}$ réactive,...). Nous ne les abordons pas de manière détaillée dans ce travail car nous avons pris le parti d'analyser trois indicateurs qui nous semblent pertinents.

\subsection{Limites de notre méta-analyse}

Nous avons identifié différentes limites à notre travail.

\subsubsection{Biais de sélection}

\section{Faible quantité d'études sélectionnées et faible échantillon total}

Suite à nos diverses étapes de tri des articles, le nombre d'études sélectionnées est au final faible. Cela peut s'expliquer par le fait que nos critères d'inclusion et d'exclusion se devaient d'être complets et précis afin de réaliser notre travail de manière ciblée. Cependant, peu d'articles possédaient ces critères (type de sport, protocoles, indicateurs) pour les intégrer à notre méta-analyse. À cela s'ajoute un nombre de participants analysé dans chaque étude également faible. Nous pensons que cela peut s'expliquer par une difficulté certaine d'intégrer plusieurs équipes sportives dans une même étude.

Suite à ces raisons, l'échantillon total de notre travail est faible et insuffisant pour représenter la population sportive et la population globale. La validité externe de l'étude en est alors affectée et ne permet pas de généraliser nos conclusions.

\section{Population mixte}

Afin de pouvoir analyser nos différents indicateurs, nous avons intégré une étude (Rupp et al., 2011) qui comportait une population mixte (neuf femmes et treize hommes). Cette dernière a pu modifier les résultats de certains paramètres mesurés de par les différences physiologiques entre les hommes et les femmes.

\subsubsection{Biais de suivi}

Dans les quatre études sélectionnées, les patients ainsi que les thérapeutes n'étaient jamais «en aveugle» car ils avaient conscience des moyens de récupération utilisés. Concernant les examinateurs, une seule de ces études était « en aveugle » contrairement aux trois autres. 


\subsubsection{Biais d'évaluation}

DOMS

L'EVA peut varier dans les limites inférieures et supérieures. Cela peut biaiser nos résultats car l'évaluation ne se fait pas de manière similaire. L'utilisation de la Standard Mean Difference dans nos forest plots nous a permis d'atténuer ces différences.

Malgré l'objectivation des courbatures par l'EVA, le ressenti des courbatures reste une notion subjective propre à chacun. Le type, l'intensité et la durée de l'exercice sont des facteurs influençant également le score des DOMS.

Dans l'étude d'Ascensão et al. (2011), les valeurs des DOMS ont été récoltées par groupes musculaires des membres inférieurs. Nous avons décidé de prendre uniquement les mesures des courbatures des quadriceps, car il n'y avait pas de données globales pour le ressenti des DOMS. Ce choix a été établi en analysant les valeurs des DOMS des différents groupes musculaires et en prenant en compte les résultats les plus élevés. Dans les autres études, elles ont été prises dans leurs globalités. Cela peut donc interférer dans la prise chiffrée de ces mesures et s'avérer être un biais pour notre métaanalyse.

\section{Capacité fonctionnelle des membres inférieurs}

Dans les études sélectionnées, nous avons remarqué que les instruments de mesures variaient pour déterminer la hauteur de saut vertical. Les différences entre les protocoles et dans les exécutions du mouvement, ainsi que les erreurs liées à la prise de mesures (participants, examinateurs et appareillages) peuvent modifier les résultats. En effet, l'article de Rupp et al. (2011) évalue la hauteur de saut par un test de saut Vertical Jump Stand qui est moins précis que le $S J$ et le $C M J$ standardisés et utilisés dans les autres études. Ce test met en jeu d'autres composantes comme la mobilité articulaire et le recrutement musculaire des membres supérieurs, ainsi que des capacités de coordination.

La capacité fonctionnelle des membres inférieurs est évaluée à la fois par les hauteurs de saut en $S J$ et en $C M J$. Les hauteurs des résultats entre ces deux sauts peuvent comporter des différences dues à la composante plyométrique du CMJ. Ces valeurs ont 
été rassemblées et les différences ont été calculées avec la Standard Mean Difference dans nos forest plots. Malgré cela, un risque de biais existe.

\subsubsection{Autres biais de notre travail}

\section{Différents exercices pré-interventions et protocoles d'immersion}

Dans les différentes études sélectionnées, les exercices pré-interventions variaient considérablement. Cela a pu engendrer des différences conséquentes dans nos résultats car les dommages musculaires provoqués ainsi que la fatigue induite n'étaient pas similaires. Il est important de mentionner que ces exercices ne sont pas, dans chaque étude, spécifiques à la pratique de sports acycliques. Dès lors, ils ne sont pas forcément représentatifs des efforts fournis dans ce type de sports.

Les protocoles d'immersion présents dans notre revue diffèrent légèrement en termes de température et de durée. Cela peut s'expliquer par le manque de consensus dans la littérature scientifique quant à un protocole d'immersion standardisé.

\section{Paramètres importants influençant la récupération non pris en compte}

Il est évident que d'autres facteurs peuvent influencer la récupération des sujets comme par exemple : la nourriture, l'environnement, l'entraînement, les qualités physiques propres à chacun ou encore la génétique. Il est difficile de contrôler ces facteurs et d'en mesurer l'impact précis sur les différentes modalités de récupération.

\section{Analyse des données}

Lors de l'utilisation des données des participants envoyées par les auteurs, nous avons constaté que certains sujets n'avaient pas de valeurs chiffrées. Nous ne les avons pas pris en compte, ce qui nous a fait perdre quelques participants dans les résultats.

Nous avons essayé d'obtenir les valeurs de chaque participant afin de calculer la moyenne et l'écart-type de la variation des indicateurs pré- et post-exercices dans chaque étude sélectionnée. Malgré nos efforts, nous n'avons pas pu obtenir des auteurs toutes les données utiles pour ces calculs. Nous avons comparé uniquement les valeurs à 24H sans tenir compte des valeurs de base. Cela a certainement biaisé certains de nos résultats. En effet, les seuls résultats à $24 \mathrm{H}$ ne sont pas représentatifs de la diminution ou de l'augmentation de l'indicateur évalué par rapport aux valeurs de base. La prise en 
considération de cette variation nous aurait permis de niveler les différences pré- et post-effort.

De part les diverses modalités de récupération ainsi que par les indicateurs analysés, nous avons, dans la majorité de nos forest plots, plusieurs comparaisons entre deux études. Cela peut rapidement influencer les résultats de manière positive ou négative.

Les indicateurs observés dans les quatre études sont mesurés à $24 \mathrm{H}$ post-effort. Il serait nécessaire de les évaluer à différents moments pour avoir une observation plus précise en termes de durée de récupération. C'est le cas, notamment, pour les DOMS qui apparaissent 12 à 48 heures après un effort musculaire intense à prédominance excentrique et pouvant persister jusqu'à 10 jours (Coudreuse et al., 2004). Cela n'est pas complètement représentatif par rapport à la durée totale possible des DOMS.

\section{Capacité fonctionnelle des membres inférieurs}

En ce qui concerne les données analysées pour la capacité fonctionnelle des membres inférieurs, un auteur ne nous les a pas fournies malgré nos demandes et elles n'ont pas pu être analysées de manière complète.

\subsection{Forces de notre revue Spécificité}

La précision des critères d'inclusion et d'exclusion représente également une force de notre étude car de ce fait, elle devient très pertinente pour la récupération par immersion dans des sports de type acyclique. En effet, les quatre études possèdent des sujets pratiquants des sports du même type ayant des contraintes similaires (football, rugby, volleyball). De plus, la moyenne d'âge des participants entre les études sélectionnées reste sensiblement similaire ( \pm 20.7 ans).

Les mesures prises pour créer nos forest plots ont été sélectionnées à $24 \mathrm{H}$ post-effort. Cela donne une indication très précise sur la récupération à court terme sur les $D O M S$, la $\mathrm{CK}$ et la hauteur de saut vertical. 


\section{Méthodologie}

Même si nous n'avons pas considéré toutes les bases de données existantes, nous estimons que les quatre utilisées (Pubmed, CINAHL, PEDro et Web of Science) ainsi que les recherches dans la littérature grise nous ont permis de réunir un nombre d'articles conséquents sur le thème de la récupération par immersion. De plus, nous avons effectué, à six mois d'intervalle, deux recherches dans la littérature afin d'intégrer dans notre méta-analyse les derniers parutions. Un autre avantage pour la recherche d'articles est notre plurilinguisme. En effet, nous avions la possibilité de sélectionner et d'évaluer des articles dans quatre langues différentes (anglais, français, allemand et italien) et nous avons même pu correspondre de manière plus aisée avec les différents auteurs afin d'obtenir des informations et des données précises. Nous estimons ainsi avoir effectué une méthodologie de recherche, de sélection et d'évaluation pertinente.

Un autre élément important pour l'élaboration de notre travail fut la possibilité d'utiliser les compétences propres à chacun et également de profiter d'un travail en équipe. Effectivement, il y a eu une alternance entre le travail solitaire et celui en binôme dans les différentes phases du projet. Lors du tri des articles ou lors de l'évaluation de la qualité des études sélectionnées, nous avons travaillé séparément avant de nous réunir pour une mise en commun. L'écriture de la discussion s'est faite conjointement afin d'échanger nos idées et d'optimiser l'exécution de cette tâche. De plus, le travail en binôme a permis une relecture systématique des différents chapitres élaborés. Ce facteur a minimisé les erreurs humaines qui peuvent survenir lors de la réalisation d'un tel projet.

\section{Précision des données utilisées et calcul de puissance statistique}

Nous avons réussi à obtenir les données chiffrées des résultats des différents articles sélectionnés grâce aux auteurs. Nous avons ainsi compilé de manière précise dans les forest plots les moyennes ainsi que les écart-types. Nous ne nous sommes jamais contentés de prendre en compte les valeurs représentées sous forme de graphiques. Les résultats de notre revue sont plus précis.

\section{Design et qualité des études}

Pour notre méta-analyse, quatre études randomisées contrôlées parues récemment ont été sélectionnées. Ces dernières sont au sommet de la pyramide de l'évidence et 
permettent d'augmenter le niveau de preuve de notre travail. De plus, toutes les études ont un score égal ou plus grand que 5 sur 8 sur l'échelle PEDro. Le design des études sélectionnées ainsi que leur qualité méthodologique permettent d'augmenter le niveau de preuve de notre revue.

\subsection{Autres travaux publiés}

Dans la littérature, nous retrouvons quelques revues systématiques et méta-analyses sur la $C W I$ et sur la $C W T$.

\subsubsection{Méta-analyse}

Bieuzen, Bleakley, \& Costello, (2013)

Ils ont évalué les effets de la $C W T$ sur dix-huit études comprenant une population mixte pratiquant tous types de sports. Les résultats pertinents pour notre travail sont les suivants :

-L'utilisation de la $C W T$ par rapport à la $P A S$ montre :

- une différence statistiquement significative en faveur de la CWT pour la réduction des $D O M S$ à $<6 \mathrm{H}, 24 \mathrm{H}, 48 \mathrm{H}, 72 \mathrm{H}$ et $>$ à $96 \mathrm{H}$,

- une limitation statistiquement significative de l'augmentation du taux de CK en faveur de la $C W T$ à $48 \mathrm{H}$ et $72 \mathrm{H}$,

- aucune différence statistiquement significative pour la puissance musculaire.

-L'utilisation de la $C W I$ par rapport à la $C W T$ indique, entre < $6 \mathrm{H}$ et $>96 \mathrm{H}$ :

- aucune différence statistiquement significative pour les DOMS,

- une tendance en faveur de la $C W I$ pour la réduction du taux de CK,

- aucune différence statistiquement significative pour la puissance musculaire.

Les auteurs concluent que l'utilisation de la $C W T$ est meilleure que la récupération passive et qu'il semble avoir une légère différence entre la $C W T$ et les autres modalités populaires de récupération (Bieuzen, Bleakley, \& Costello, 2013). 


\section{Leeder et al. (2012)}

Ils ont analysé dans quatorze études les effets de la $C W I$ dans une population mixte sportive et non-sportive. Les résultats révèlent par rapport à un groupe contrôle que la $C W I$ est effective dans la limitation des DOMS jusqu'à $96 \mathrm{H}$ post-effort, de même que pour la puissance musculaire à $24 \mathrm{H}, 48 \mathrm{H}$ et $72 \mathrm{H}$. Pour la $\mathrm{CK}$, l'utilisation de la $C W I$ montre une différence statistiquement significative à $24 \mathrm{H}, 48 \mathrm{H}$ et $72 \mathrm{H}$.

En conclusion, cette méta-analyse indique que la $C W I$ est une stratégie efficace dans la réduction des DOMS, même si le mécanisme reste à comprendre (Leeder et al., 2012).

\section{Diong \& Kamper (2013)}

Une mise à jour d'une revue systématique de Bleakley et al. (2012) a été effectuée dernièrement par Diong \& Kamper (2013). Ils ont analysé dix-sept études afin de déterminer les effets de la $C W I$ sur la prévention des courbatures post-efforts. Les résultats démontrent une différence statistiquement significative en faveur de la $C W I$ pour les $D O M S$ à $24 \mathrm{H}, 48 \mathrm{H}, 72 \mathrm{H}$ et $96 \mathrm{H}$ par rapport à la $P A S$. Aucune différence n'a été retrouvée entre la $C W I$ et la $C W T$.

Cette revue fournit certains éléments de preuve quant à la réduction des courbatures post-exercice par la $C W I$ en comparaison de la $P A S$. Ces résultats sont à interpréter avec prudence en raison de la faible qualité des études et de la taille de l'effet (Diong \& Kamper, 2013). 


\section{Conclusion}

\subsection{Implications pour la pratique}

Notre revue systématique et la méta-analyse réalisée nous permettent d'affirmer que l'utilisation de la $C W I$ dans l'optique de réduire les valeurs des DOMS à $24 \mathrm{H}$ chez des sportifs acycliques est statistiquement significative. L'utilisation de la $C W I$ permet de limiter cliniquement l'élévation du taux de CK à $24 \mathrm{H}$ post-effort. En ce qui concerne la capacité fonctionnelle des membres inférieurs, l'utilisation de la $C W I$ ou de la $C W T$ ne représente aucun intérêt à $24 \mathrm{H}$ post-effort. Il faut considérer ces résultats avec prudence par rapport au faible échantillon analysé et la présence de certains biais.

En transférant cette conclusion à la physiothérapie dans des milieux sportifs, la $C W I$ s'avère être performante pour atténuer les DOMS et le taux de $\mathrm{CK}$, ce qui peut améliorer la récupération suite à un effort musculaire.

\subsection{Recherches futures}

Suites à nos résultats, d'autres recherches sont nécessaires afin d'évaluer les effets de l'immersion en terme de récupération. Il faudrait effectuer des essais randomisés contrôlés de haute qualité méthodologique chez des sportifs acycliques en incluant un nombre de participants plus élevé. Il serait intéressant de conduire des études en suivant des équipes sur plusieurs mois afin d'évaluer les modalités d'immersion sur le long terme. Toutes ces études permettraient peut-être de trouver un consensus en faveur d'un des ces moyens de récupération.

Dès lors, les études devraient se consacrer à la recherche d'un protocole standardisé quant à la durée, à la température et à la hauteur de l'immersion. Finalement, une nouvelle méta-analyse permettrait de rassembler toutes les études et éventuellement de généraliser sur ce moyen de récupération. 


\subsection{Apports personnels}

La réalisation de ce travail de Bachelor nous a permis d'enrichir nos connaissances théoriques sur le thème abordé mais également de les confronter à l'évidence scientifique. Cette dernière, par la consultation d'articles, permet d'actualiser régulièrement nos savoirs et de rester à la pointe des informations concernant notre pratique en physiothérapie. De surcroît, nous sommes désormais capables de lire de manière critique les études présentes dans la littérature et de les interpréter. Une progression a été observée dans un certain nombre d'aspects de la communication notamment dans l'écoute attentive des autres, l'échange d'idées et l'argumentation de ses choix. Une organisation structurée ainsi qu'une rigueur et une précision dans la méthodologie ont été nécessaires et acquises pour le bon déroulement de ce projet.

Tous ces éléments nous ont permis de développer et d'acquérir différentes qualités nécessaires au métier de physiothérapeute que nous appliquerons prochainement. 


\section{Références}

Armstrong, R. B., Warren, G. L., \& Warren, J. A. (1991). Mechanisms of exercise-induced muscle fibre injury. Sports medicine (Auckland, N.Z.), 12(3), 184-207.

Ascensão, A., Leite, M., Rebelo, A. N., Magalhäes, S., \& Magalhäes, J. (2011). Effects of cold water immersion on the recovery of physical performance and muscle damage following a one-off soccer match. Journal of Sports Sciences, 29(3), 217-225.

doi:10.1080/02640414.2010.526132

Bailey, D. M., Erith, S. J., Griffin, P. J., Dowson, A., Brewer, D. S., Gant, N., \& Williams, C. (2007). Influence of cold-water immersion on indices of muscle damage following prolonged intermittent shuttle running. Journal of sports sciences, 25(11), 1163-1170. doi:10.1080/02640410600982659

Baird, M. F., Graham, S. M., Baker, J. S., \& Bickerstaff, G. F. (2012). Creatine-Kinase- and Exercise-Related Muscle Damage Implications for Muscle Performance and Recovery. Journal of Nutrition and Metabolism, 2012, 1-13. doi:10.1155/2012/960363

Barnett, A. (2006). Using recovery modalities between training sessions in elite athletes: does it help? Sports medicine (Auckland, N.Z.), 36(9), 781-796.

Biddle, S., \& Mutrie, N. (2001). Psychology of Physical Activity: Determinants, Well-being, and Interventions. Routledge.

Bieuzen, F., Bleakley, C. M., \& Costello, J. T. (2013). Contrast water therapy and exercise induced muscle damage: a systematic review and meta-analysis. PloS one, 8(4), e62356. doi:10.1371/journal.pone.0062356

Bleakley, C. M., \& Davison, G. W. (2010). What is the biochemical and physiological rationale for using cold-water immersion in sports recovery? A systematic review. British journal of sports medicine, 44(3), 179-187. doi:10.1136/bjsm.2009.065565

Bleakley, C., McDonough, S., Gardner, E., Baxter, G. D., Hopkins, J. T., \& Davison, G. W. (2012). Cold-water immersion (cryotherapy) for preventing and treating muscle soreness after exercise. In The Cochrane Collaboration \& C. Bleakley (Éd.), Cochrane Database of Systematic Reviews. Chichester, UK: John Wiley \& Sons, Ltd. Consulté à 
l'adresse http://summaries.cochrane.org/CD008262/cold-water-immersion-forpreventing-and-treating-muscle-soreness-after-exercise

Bobbert, M F, Gerritsen, K. G., Litjens, M. C., \& Van Soest, A. J. (1996). Why is countermovement jump height greater than squat jump height? Medicine and science in sports and exercise, 28(11), 1402-1412.

Bobbert, Maarten F, \& Casius, L. J. R. (2005). Is the effect of a countermovement on jump height due to active state development? Medicine and science in sports and exercise, 37(3), 440-446.

Bompa, T. O., \& Haff, G. G. (2009). Periodization: Theory and Methodology of Training. Human Kinetics.

Borges, O., \& Essén-Gustavsson, B. (1989). Enzyme activities in type I and II muscle fibres of human skeletal muscle in relation to age and torque development. Acta physiologica Scandinavica, 136(1), 29-36.

Brancaccio, P., Maffulli, N., \& Limongelli, F. M. (2007). Creatine kinase monitoring in sport medicine. British medical bulletin, 81-82, 209-230. doi:10.1093/bmb/ldm014

Brukner, P., (D.R.C.O.G.), P. B., \& Khan, K. (2001). Clinical sports medicine. McGraw-Hill. Buckthorpe, M., Morris, J., \& Folland, J. P. (2012). Validity of vertical jump measurement devices. Journal of sports sciences, 30(1), 63-69. doi:10.1080/02640414.2011.624539

Caspersen, C. J., Powell, K. E., \& Christenson, G. M. (1985). Physical activity, exercise, and physical fitness: definitions and distinctions for health-related research. Public Health Reports, 100(2), 126-131.

Castagna, C., Ganzetti, M., Ditroilo, M., Giovannelli, M., Rocchetti, A., \& Manzi, V. (2013). Concurrent validity of vertical jump performance assessment systems. Journal of strength and conditioning research / National Strength \& Conditioning Association, 27(3), 761-768. doi:10.1519/JSC.0b013e31825dbcc5

Cochrane, D. J. (2004). Alternating hot and cold water immersion for athlete recovery: a review. Physical Therapy in Sport, 5(1), 26-32. doi:10.1016/j.ptsp.2003.10.002

Coffey, V., Leveritt, M., \& Gill, N. (2004). Effect of recovery modality on 4-hour repeated treadmill running performance and changes in physiological variables. Journal of science and medicine in sport / Sports Medicine Australia, 7(1), 1-10. 
Cometti, G. (1997, avril). La pliométrie. Revue EPS (éducation physique et sport), (264), 39-50.

Cometti, G., \& Cometti, D. (2006). La pliométrie : Méthodes, entraînements et exercices. Chiron. Commission médicale du Comité Internationale Olympique. (2008). Physiothérapie du sport: soins - prévention - récupération. Comité Internationale Olympique. Consulté le 01 juin 2013 à l'adresse http://www.olympic.org/fr/commission-medicale?tab=livres-etdocuments

Coudreuse, J. ., Dupont, P., \& Nicol, C. (2004). Douleurs musculaires posteffort. Annales de Réadaptation et de Médecine Physique, 47(6), 290-298. doi:10.1016/j.annrmp.2004.05.012

Crewther, B. T., Kilduff, L. P., Cunningham, D., Cook, C., \& Yang, G.-Z. (2010). Validity of two kinematic systems for calculating force and power during squat jumps. British Journal of Sports Medicine, 44(14), i26-i26. doi:10.1136/bjsm.2010.078972.79

De Nardi, M., La Torre, A., Barassi, A., Ricci, C., \& Banfi, G. (2011). Effects of cold-water immersion and contrast-water therapy after training in young soccer players. The Journal of Sports Medicine and Physical Fitness, 51(4), 609-615.

Delextrat, A., Calleja-González, J., Hippocrate, A., \& Clarke, N. D. (2012). Effects of sports massage and intermittent cold-water immersion on recovery from matches by basketball players. Journal of sports sciences. doi:10.1080/02640414.2012.719241

Diong, J., \& Kamper, S. J. (2013). Cold water immersion (cryotherapy) for preventing muscle soreness after exercise. British journal of sports medicine. doi:10.1136/bjsports-2013092433

Efstratiadis, G., Voulgaridou, A., Nikiforou, D., Kyventidis, A., Kourkouni, E., \& Vergoulas, G. (2007). Rhabdomyolysis updated. Hippokratia, 11(3), 129-137.

Ehlers, G. G., Ball, T. E., \& Liston, L. (2002). Creatine Kinase Levels are Elevated During 2-ADay Practices in Collegiate Football Players. Journal of Athletic Training, 37(2), $151-156$.

Elias $^{1}$, G. P., Varley, M. C., Wyckelsma, V. L., McKenna, M. J., Minahan, C. L., \& Aughey, R. J. (2012). Effects of water immersion on posttraining recovery in Australian footballers. International journal of sports physiology and performance, 7(4), 357-366. 
Elias $^{2}$, G. P., Wyckelsma, V. L., Varley, M. C., McKenna, M. J., \& Aughey, R. J. (2012). Effectiveness of Water Immersion on Post-Match Recovery in Elite Professional Footballers. International journal of sports physiology and performance.

Eston, R., \& Peters, D. (1999). Effects of cold water immersion on the symptoms of exerciseinduced muscle damage. Journal of sports sciences, 17(3), 231-238. doi:10.1080/026404199366136

Faculté de Médecine Pierre et Marie Curie. Réserves énergétiqes - Créatine Phosphokinase. Informations médicales. Consulté le 01 juin 2013 à l'adresse http://www.chups.jussieu.fr/polys/biochimie/REbioch/POLY.Chp.2.4.html

Faulkner, J. A., Brooks, S. V., \& Opiteck, J. A. (1993). Injury to skeletal muscle fibers during contractions: conditions of occurrence and prevention. Physical therapy, 73(12), 911-921.

Gagliano, M., Corona, D., Giuffrida, G., Giaquinta, A., Tallarita, T., Zerbo, D., ... Veroux, M. (2009). Low-intensity body building exercise induced rhabdomyolysis: a case report. Cases journal, 2(1), 7. doi:10.1186/1757-1626-2-7

Gerodimos, V., Zafeiridis, A., Perkos, S., Dipla, K., Manou, V., \& Kellis, S. (2008). The contribution of stretch-shortening cycle and arm-swing to vertical jumping performance in children, adolescents, and adult basketball players. Pediatric exercise science, 20(4), 379-389.

Gill, N. D., Beaven, C. M., \& Cook, C. (2006). Effectiveness of post-match recovery strategies in rugby players. British journal of sports medicine, 40(3), 260-263. doi:10.1136/bjsm.2005.022483

Goodall, S., \& Howatson, G. (2008). The effects of multiple cold water immersions on indices of muscle damage.(Research article)(Clinical report). Journal of Sports Science and Medicine. Consulté à l'adresse http://www.highbeam.com/doc/1G1-180029919.html

Gregson, W., Black, M. A., Jones, H., Milson, J., Morton, J., Dawson, B., ... Green, D. J. (2011). Influence of cold water immersion on limb and cutaneous blood flow at rest. The American journal of sports medicine, 39(6), 1316-1323.

doi:10.1177/0363546510395497 
Guezennec, Charles-Yannick. (s. d.). Aspects physiologiques de la récupération.

Essonne.franceolympique. Consulté 1 juillet 2012, à l'adresse

http://essonne.franceolympique.com/essonne/fichiers/File/larecuperation.pdf

Hausswirth, C., Bieuzen, F., Barbiche, E., \& Brisswalter, J. (2010). Réponses physiologiques liées à une immersion en eau froide et à une cryostimulation-cryothérapie en corps entier : effets sur la récupération après un exercice musculaire. Science \& Sports, 25(3), 121-131. doi:10.1016/j.scispo.2009.12.001

Hortobágyi, T., \& Denahan, T. (1989). Variability in creatine kinase: methodological, exercise, and clinically related factors. International journal of sports medicine, 10(2), 69-80.

Howard G. Knuttgen, W. J. K. (1987). Terminology and Measurement in Exercise Performance. The Journal of Strength \&amp; Conditioning Research, 1(1), 1-10.

Huerta-Alardín, A. L., Varon, J., \& Marik, P. E. (2005). Bench-to-bedside review:

Rhabdomyolysis -- an overview for clinicians. Critical care (London, England), 9(2), 158-169. doi:10.1186/cc2978

Ingram, J., Dawson, B., Goodman, C., Wallman, K., \& Beilby, J. (2009). Effect of water immersion methods on post-exercise recovery from simulated team sport exercise. Journal of science and medicine in sport / Sports Medicine Australia, 12(3), 417-421. doi:10.1016/j.jsams.2007.12.011

Jastrzębski, Z. (2006). Changes of chosen blood parameters in football players in relation to applied training loads during competition. Biology of Sport, 23(1), 85-96.

Karoubi, L., Maire, P., Ouvrard, C., \& Collectif. (2008). Le petit Larousse illustré : Grand format (édition 2009.). Larousse.

Kellmann, M. (2010). Preventing overtraining in athletes in high-intensity sports and stress/recovery monitoring. Scandinavian journal of medicine \& science in sports, 20 Supp/ 2, 95-102. doi:10.1111/j.1600-0838.2010.01192.x

Kellmann, Michael, \& Kallus, K. W. (2001). Recovery-stress Questionnaire for Athletes: User Manual. Human Kinetics.

Kinugasa, T., \& Kilding, A. E. (2009). A comparison of post-match recovery strategies in youth soccer players. Journal of strength and conditioning research / National Strength \& Conditioning Association, 23(5), 1402-1407. doi:10.1519/JSC.0b013e3181a0226a 
Klavora, P. (2000). Vertical-jump Tests: A Critical Review. Strength \&amp; Conditioning Journal, 22(5), 70.

Kniffki, K. D., Mense, S., \& Schmidt, R. F. (1978). Responses of group IV afferent units from skeletal muscle to stretch, contraction and chemical stimulation. Experimental brain research. Experimentelle Hirnforschung. Expérimentation cérébrale, 31(4), 511-522.

Komi, P. V. (2000). Stretch-shortening cycle: a powerful model to study normal and fatigued muscle. Journal of biomechanics, 33(10), 1197-1206.

Komi, P. V., \& Gollhofer, A. (1997). Stretch reflexes can have an important role in force enhancement during SSC exercise. Journal of Applied Biomechanics, 13, 451-460.

Lane, K. N., \& Wenger, H. A. (2004). Effect of selected recovery conditions on performance of repeated bouts of intermittent cycling separated by 24 hours. Journal of strength and conditioning research / National Strength \& Conditioning Association, 18(4), 855-860. doi:10.1519/14183.1

Latham, J., Campbell, D., Nichols, W., \& Mott, T. (2008). Clinical inquiries. How much can exercise raise creatine kinase level--and does it matter? The Journal of family practice, 57(8), 545-547.

Leeder, J., Gissane, C., van Someren, K., Gregson, W., \& Howatson, G. (2012). Cold water immersion and recovery from strenuous exercise: a meta-analysis. British Journal of Sports Medicine, 46(4), 233-240. doi:10.1136/bjsports-2011-090061

Low, J., Reed, A., \& (SRP.), A. R. (2000). Electrotherapy Explained: Principles and Practice. Elsevier Health Sciences.

Maher, C. G., Sherrington, C., Herbert, R. D., Moseley, A. M., \& Elkins, M. (2003). Reliability of the PEDro scale for rating quality of randomized controlled trials. Physical therapy, 83(8), $713-721$.

Malkinson, T. J., Martin, S., Simper, P., \& Cooper, K. E. (1981). Expired air volumes of males and females during cold water immersion. Canadian journal of physiology and pharmacology, 59(8), 843-846.

Malliou, P., Ispirlidis, I., Beneka, A., Taxildaris, K., \& Godolias, G. (2003). Vertical jump and knee extensors isokinetic performance in professional soccer players related to the phase of the training period. Isokinetics and Exercise Science, 11(3), 165-169. 
Mantoni, T., Belhage, B., Pedersen, L. M., \& Pott, F. C. (2007). Reduced cerebral perfusion on sudden immersion in ice water: a possible cause of drowning. Aviation, space, and environmental medicine, 78(4), 374-376.

McBride, J. M., McCaulley, G. O., \& Cormie, P. (2008). Influence of preactivity and eccentric muscle activity on concentric performance during vertical jumping. Journal of strength and conditioning research / National Strength \& Conditioning Association, 22(3), 750-757. doi:10.1519/JSC.0b013e31816a83ef

McCaulley, G. O., Cormie, P., Cavill, M. J., Nuzzo, J. L., Urbiztondo, Z. G., \& McBride, J. M. (2007). Mechanical efficiency during repetitive vertical jumping. European journal of applied physiology, 101(1), 115-123. doi:10.1007/s00421-007-0480-1

Monod, H., Flandrois, R., \& Vandewalle, H. (2011). Physiologie Du Sport. Elsevier Masson.

Monod, H., \& Rochcongar, P. (2009). Médecine du sport. Elsevier Masson.

N. Marieb. Elaine, \& Hoehn, Katja. (2010). Anatomie et physiologie humaines. Pearson.

Newton, R. U., \& Kraemer, W. J. (1994). Developing explosive muscular power: Implications for a mixed methods training strategy. Strength and Conditioning, 16, 20-20.

Norkowski, H. (2002). Anaerobic Power of Handball Players Representing Various Sport Levels. Journal of Human Kinetics, 7, 43-50.

Pilardeau, P. (1995). Biochimie et nutrition des activités physiques et sportives: Le métabolisme énergétique. Masson.

Pointon, M., \& Duffield, R. (2012). Cold water immersion recovery after simulated collision sport exercise. Medicine and Science in Sports and Exercise, 44(2), 206-216. doi:10.1249/MSS.0b013e31822b0977

Pointon, M., Duffield, R., Cannon, J., \& Marino, F. E. (2011). Cold water immersion recovery following intermittent-sprint exercise in the heat. European Journal of Applied Physiology. doi:10.1007/s00421-011-2218-3

Pournot, H., Bieuzen, F., Duffield, R., Lepretre, P.-M., Cozzolino, C., \& Hausswirth, C. (2011). Short term effects of various water immersions on recovery from exhaustive intermittent exercise. European Journal of Applied Physiology, 111(7), 1287-1295. doi:10.1007/s00421-010-1754-6 
Prelle, A., Tancredi, L., Sciacco, M., Chiveri, L., Comi, G. P., Battistel, A., ... Moggio, M. (2002). Retrospective study of a large population of patients with asymptomatic or minimally symptomatic raised serum creatine kinase levels. Journal of neurology, 249(3), 305-311.

Prentice, W. E. (1999). Therapeutic modalities in sports medicine. WCB/McGraw-Hill.

Reilly, T., \& Ekblom, B. (2005). The use of recovery methods post-exercise. Journal of sports sciences, 23(6), 619-627. doi:10.1080/02640410400021302

Rowsell, G. J., Coutts, A. J., Reaburn, P., \& Hill-Haas, S. (2009). Effects of cold-water immersion on physical performance between successive matches in high-performance junior male soccer players. Journal of sports sciences, 27(6), 565-573. doi:10.1080/02640410802603855

Rowsell, G. J., Coutts, A. J., Reaburn, P., \& Hill-Haas, S. (2011). Effect of post-match coldwater immersion on subsequent match running performance in junior soccer players during tournament play. Journal of sports sciences, 29(1), 1-6. doi:10.1080/02640414.2010.512640

Rupp, K. A., Selkow, N. M., Parente, W. R., Ingersoll, C. D., Weltman, A. L., \& Saliba, S. A. (2011). The Effect of Cold Water Immersion on 48 Hour Performance Testing in Collegiate Soccer Players.: Exercise and Sport Injury Laboratory, University of Virginia. Journal of Strength and Conditioning Research / National Strength \& Conditioning Association. doi:10.1519/JSC.0b013e318239c3a1

Scherrer, J., \& Monod, H. (1960). Le travail musculaire local et la fatigue chez l'homme. The Journal of Physiology, 52, 419-501.

Sellwood, K. L., Brukner, P., Williams, D., Nicol, A., \& Hinman, R. (2007). Ice-water immersion and delayed-onset muscle soreness: a randomised controlled trial. British journal of sports medicine, 41(6), 392-397. doi:10.1136/bjsm.2006.033985

Signorile, J. F., Ingalls, C., \& Tremblay, L. M. (1993). The effects of active and passive recovery on short-term, high intensity power output. Canadian journal of applied physiology = Revue canadienne de physiologie appliquée, 18(1), 31-42.

Smith, L. L. (1991). Acute inflammation: the underlying mechanism in delayed onset muscle soreness? Medicine and science in sports and exercise, 23(5), 542-551. 
Takarada, Y. (2003). Evaluation of muscle damage after a rugby match with special reference to tackle plays. British Journal of Sports Medicine, 37(5), 416-419. doi:10.1136/bjsm.37.5.416

The Cochrane Collaboration. (2011). Cochrane Handbook for Systematic Reviews of Interventions (Vol. Version 5.1.0). Higgins JPT, Green S. Consulté à l'adresse www.cochrane-handbook.org

Thompson. (2011, juillet). What Form of Power Is Needed In Acyclic \& Cyclic Sports? eHow. Consulté 1 juillet 2012, à l'adresse http://www.ehow.com/info_8756191_form-neededacyclic-cyclic-sports.html

Tietz, N. W., Shuey, D. F., \& Wekstein, D. R. (1992). Laboratory values in fit aging individuals-sexagenarians through centenarians. Clinical chemistry, 38(6), 1167-1185.

Vaile, J, Halson, S., Gill, N., \& Dawson, B. (2008). Effect of hydrotherapy on recovery from fatigue. International journal of sports medicine, 29(7), 539-544. doi:10.1055/s-2007989267

Vaile, J, O'Hagan, C., Stefanovic, B., Walker, M., Gill, N., \& Askew, C. D. (2011). Effect of cold water immersion on repeated cycling performance and limb blood flow. British journal of sports medicine, 45(10), 825-829. doi:10.1136/bjsm.2009.067272

Vaile, Joanna, Halson, S., Gill, N., \& Dawson, B. (2008). Effect of hydrotherapy on the signs and symptoms of delayed onset muscle soreness. European journal of applied physiology, 102(4), 447-455. doi:10.1007/s00421-007-0605-6

Van Ingen Schenau, G. J., Bobbert, M. F., \& De Haan, A. (1997). Does elastic energy enhance work and efficiency in the stretch-shortening cycle? Journal of Applied Biomechanics, $13,389-415$.

Van Wyk, D., \& Lambert, M. (2009). Recovery strategies implemented by sport support staff of elite rugby players in South Africa. South African Journal of Physiotherapy, 65(1), 41-46.

Wisloff, U., Castagna, C., Helgerud, J., Jones, R., \& Hoff, J. (2004). Strong correlation of maximal squat strength with sprint performance and vertical jump height in elite soccer players. British Journal of Sports Medicine, 38(3), 285-288.

doi:10.1136/bjsm.2002.002071 
Yamane, M., Teruya, H., Nakano, M., Ogai, R., Ohnishi, N., \& Kosaka, M. (2006). Post-exercise leg and forearm flexor muscle cooling in humans attenuates endurance and resistance training effects on muscle performance and on circulatory adaptation. European journal of applied physiology, 96(5), 572-580. doi:10.1007/s00421-005-0095-3

Zuluaga, M. (1995). Sports Physiotherapy: Applied Science and Practice. Churchill Livingstone. 


\section{Liste des illustrations et des tableaux}

\subsection{Illustrations}

Figure 1 : transformation de l'ADP en ATP par l'action de la CK sur la Créatine-P............11

Figure 2 : fluxogramme de la sélection des articles.................................... 20

\subsection{Tableaux}

Tableau 2 : évaluation de la qualité des articles grâce à l'échelle PEDro...................... 21

Tableau 2 : données des études incluses............................................. 23

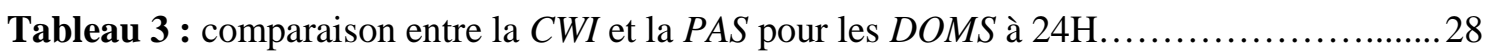

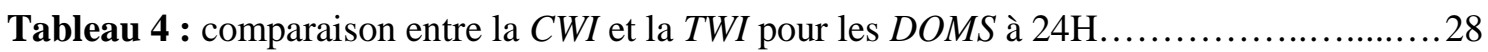

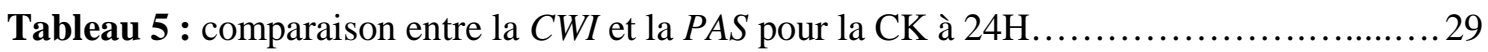

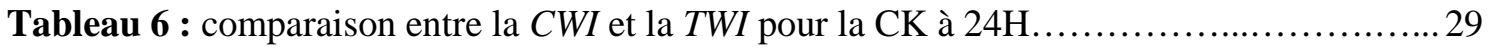

Tableau 7 : comparaison entre la $C W I$ et la $P A S$ pour la hauteur de saut à $24 \mathrm{H} \ldots \ldots \ldots \ldots \ldots \ldots . . . . . . .30$

Tableau 8 : comparaison entre la $C W I$ et la $T W I$ pour la hauteur de saut à $24 \mathrm{H} \ldots \ldots \ldots \ldots \ldots . \ldots . \ldots . \ldots$

Tableau 9 : synthèse des résultats obtenus............................................. 31 


\section{Annexes}

\section{Annexe I - Équations de recherche}

\section{PubMed}

- "Sports"[Mesh] AND "Hydrotherapy"[Mesh] OR "waterimmersion" OR "water immersion" OR "water-immersion" OR "contrast water immersion" OR "ice immersion" OR "cold water immersion" OR "cold immersion" OR "cold water immersion therapy" OR "water therapy" OR "contrast water immersion" OR "contrast water therapy" AND ("Recovery of Function"[Mesh] OR "Convalescence"[Mesh] OR "Rehabilitation"[Mesh] OR "Elasticity"[Mesh] OR "jump" OR "creatine kinase" OR "muscle soreness" OR "DOMS" OR "DOMS muscle" OR "muscle pain" OR "delayed onset muscle soreness" OR "life quality" $O R$ "quality of life" $O R$ "recovery" OR "recuperation" OR "post competition" OR "postcompetition" OR "post-competition" OR "postexercise" OR "post-exercise" OR "post exercise" OR "post effort" OR "post-effort" OR "posteffort"

\section{CINAHL}

- "cryotherapy OR "waterimmersion" OR "water immersion" OR "waterimmersion" OR "contrast water immersion" OR "ice immersion" OR "cold water immersion" OR "cold immersion" OR "cold water immersion therapy" OR "water therapy" OR "contrast water immersion" OR "contrast water therapy") AND ("jump" OR "creatine kinase" OR "muscle soreness" OR "DOMS" OR "DOMS muscle" OR "muscle pain" OR "delayed onset muscle soreness" OR "life quality" OR"quality of life" OR "recovery" OR "recuperation" OR "post competition" OR "postcompetition" OR "post-competition" OR "postexercise" OR "post-exercise" OR "post exercise" OR "post effort" OR " post-effort" OR "posteffort") AND ("sports" OR "athlete" OR "rugby" OR "football" OR "soccer" OR "basketball" 


\section{Annexe II - Échelle PEDro}

\section{Échelle PEDro}

1. les critères d'éligibilité ont été précisés

non $\sqsupset$ oui $\square$

où:

2. les sujets ont été répartis aléatoirement dans les groupes (pour un essai croisé, l' ordre des traitements reçus par les sujets a été attribué aléatoirement)

$\begin{array}{ll}\text { non } \sqcup \text { oui } \square & \text { où: } \\ \text { non } コ \text { oui } \square & \text { où: }\end{array}$

3. la répartition a respecté une assignation secrète non $\sqcup$ oui घ où: non $\sqcup$ oui $\square$ où: non $コ$ oui $\square$ oû: non $\sqcup$ oui $\square$ où: de jugement essentiels

8. les mesures, pour au moins un des critères de jugement essentiels, ont été obtenues pour plus de $85 \%$ des sujets initialement répartis dans les groupes

non อ จ

9. tous les sujets pour lesquels les résultats étaient disponibles ont reçu le traitement ou ont suivi l' intervention contrôle conformément à leur répartition ou, quand cela n'a pas été le cas, les données d'au moins un des critères de jugement essentiels ont été analysées "en intention de traiter"

non $\sqcup$ oui $\square$ où:

10. les résultats des comparaisons statistiques intergroupes sont indiqués pour au moins un des critères de jugement essentiels

non $\sqcup$ oui $\square$ où:

11. pour au moins un des critères de jugement essentiels, l'étude indique à la fois l'estimation des effets et l'estimation de leur variabilité non $コ$ oui $コ$ où:

L'échelle PEDro est basée sur la liste Delphi développée par Verhagen et ses collègues au département d’épidémiologie de 1'Université de Maastricht (Verhagen AP et al (1998). The Delphi list: a criteria list for quality assessment of randomised clinical trials for conducting systematic reviews developed by Delphi consensus Journal of Clinical Epidemiology, $51(12): 1235-4 I)$. Cette liste est basée sur un "consensus d'experts" et non, pour la majeure partie, sur des données empiriques. Deux items supplémentaires à la liste Delphi (critères 8 et 10 de l'échelle PEDro) ont été inclus dans I'échelle PEDro. Si plus de données empiriques apparaissent, il deviendra éventuellement possible de pondérer certains critères de manière à ce que le score de PEDro reflette l'importance de chacun des items.

L'objectif de l'échelle PEDro est d' aider l'utilisateur de la base de données PEDro à rapidement identifier quels sont les essais cliniques réellement ou potentiellement randomisés indexés dans PEDro (c'est-à-dire les essais contrólés randomisés et les essais cliniques contrôlés, sans précision) qui sont susceptibles d'avoir une bonne validité inteme (critères 2 à 9), et peuvent avoir suffisamment d'informations statistiques pour rendre kurs résultats interprétables (critères 10 à 11). Un critere supplémentaire (critère 1) qui est relatif à la validité "externe" (c'est "la généralisabilité" de l'essai ou son "applicabilité") a été retenu dans l'échelle PEDro pour prendre en compte toute la liste Delphi, mais ce critère n’est pas comptabilisé pour calculer le score PEDro cité sur le site Intemet de PEDro.

L'échelle PEDro ne doit pas être utilisée pour mesurer la "validité" des conclusions d'une étude. En particulier, nous mettons en garde les utilisateurs de l'échelle PEDro sur le fait que les études qui montrent des effets significatifs du traitement et qui ont un score élevé sur l'échelle PEDro, ne signifie pas nécessairement que le traitement est cliniquement utile. II faut considérer aussi si la taille de l'effet du traitement est suffisamment grande pour que cela vaille la peine cliniquement d'appliquer le traitement. De même, il faut évaluer si le rapport entre les effets positifs du traitement et ses effets négatifs est favorable. Enfin, la dimension coûtefficacité du traitement est à prendre compte pour effectuer un choix. L'échelle ne devrait pas être utilisée pour comparer la "qualité" des essais réalisés dans différents domaines de la physiothérapie, essentiellement parce qu'il n'est pas possible de satisfaire à tous les items de cette échelle dans certains domaines de la pratique kinésithérapique.

Dernière modification le 21 juin 1999. Traduction française le 1 juillet 2010 


\section{Annexe III - Tableaux d'extraction des données}

Ascensão et al. (2011)

\begin{tabular}{|c|c|c|c|c|}
\hline \multicolumn{5}{|c|}{ DOMS (quadriceps) (0-10) } \\
\hline & Baseline & 30 min. & $24 \mathrm{H}$ & $48 \mathrm{~h}$ \\
\hline $\boldsymbol{C W I}$ (10) & 0 & $3.4 \pm 1.4$ & $3.7 \pm 1.9$ & $3.1 \pm 1.5$ \\
\hline TWI (10) & 0 & $4.1 \pm 0.9$ & $6.1 \pm 1.6$ & $4.7 \pm 1.5$ \\
\hline \multicolumn{5}{|c|}{ Créatine kinase (U/L) } \\
\hline \multicolumn{7}{|c|}{ baseline } & 30 min. & $24 \mathrm{H}$ & $48 \mathrm{~h}$ \\
\hline $\boldsymbol{C W I}$ (10) & $135+-7.7$ & $300.2+-22.4$ & $729.9+-61.5$ & $829.2+-63.9$ \\
\hline TWI (10) & $133.3+-8.5$ & $409.6+-15.5$ & $933.5+-120$ & $931.8+-48.2$ \\
\hline
\end{tabular}

\begin{tabular}{|c|c|c|c|c|}
\hline \multicolumn{5}{|c|}{ SJ $(\mathbf{c m})$} \\
\hline & Baseline & 30 min. & $24 \mathrm{H}$ & $48 \mathrm{~h}$ \\
\hline CWI (10) & $39.2+-2.9$ & - & $38.2+-5.0$ & $40.9+-5.2$ \\
\hline TWI (10) & $39.3+-3.2$ & - & $35.9+-4.3$ & $36.1+-5.6$ \\
\hline \multicolumn{5}{|c|}{ CMJ (cm) } \\
\hline & Baseline & 30 min. & $24 \mathrm{H}$ & $48 \mathrm{~h}$ \\
\hline CWI (10) & $45.3+-8.5$ & - & $38.7+-10.2$ & $41.0+-4.9$ \\
\hline TWI (10) & $45.1+-8.4$ & - & $33.2+-10.2$ & $35.3+-8.7$ \\
\hline
\end{tabular}

Bailey et al. (2007)

\begin{tabular}{|c|c|c|c|c|c|}
\hline \multicolumn{6}{|c|}{ DOMS (-10) } \\
\hline & Baseline & Post-ex & $1 \mathrm{H}$ & $24 \mathrm{H}$ & $48 \mathrm{H}$ \\
\hline CWI (10) & $1.2+-0.4$ & $6.2+-1.8$ & $3.5+-1.6$ & $4.6+-1.0$ & $4.4+-1.6$ \\
\hline PAS (10) & $1.7+-1.1$ & $6.9+-2.2$ & $5.6+-2.3$ & $6.8+-1.5$ & $5.7+-0.8$ \\
\hline
\end{tabular}

\begin{tabular}{|c|c|c|c|c|c|}
\hline \multicolumn{7}{|c|}{ CK (U/L) } \\
\hline & Baseline & Post-ex & $1 \mathrm{H}$ & $24 \mathrm{H}$ & $48 \mathrm{H}$ \\
\hline $\boldsymbol{C W I}(\mathbf{1 0})$ & $324+-257$ & $582+-381$ & $910+-410$ & $1246+-353$ & $529+-274$ \\
\hline PAS (10) & $452+-292$ & $760+-317$ & $1028+-490$ & $1358+-473$ & $766+-461$ \\
\hline
\end{tabular}

Pournot et al. (2011)

\begin{tabular}{|c|c|c|c|c|}
\hline \multicolumn{5}{|c|}{ DOMS (0-10) } \\
\hline & Pré-ex. & Post-ex & $1 \mathrm{H}$ & $24 \mathrm{H}$ \\
\hline CWI (13) & $1 \pm 2$ & & & $4.4 \pm 2.5$ \\
\hline CWT (10) & $1 \pm 2$ & & & $4.2 \pm 1.6$ \\
\hline TWI (9) & $2 \pm 2$ & & & $6.6 \pm 2.5$ \\
\hline PAS (9) & $2 \pm 1$ & & & $4.7 \pm 2.0$ \\
\hline
\end{tabular}




\begin{tabular}{|c|c|c|c|c|}
\hline \multicolumn{5}{|c|}{ CK (U/L) } \\
\hline & Pré-ex & Post-ex & $1 \mathrm{H}$ & $24 \mathrm{H}$ \\
\hline $\boldsymbol{C W I}$ (11) & $387.8 \pm 529.8$ & & $471.5 \pm 618.5$ & $408.5 \pm 357.3$ \\
\hline $\boldsymbol{C W T}$ (10) & $522.5 \pm 322.5$ & & $655.7 \pm 311.6$ & $982.5 \pm 515.4$ \\
\hline TWI (9) & $330.9 \pm 222.3$ & & $583.3 \pm 351.0$ & $834.6 \pm 492.3$ \\
\hline PAS (9) & $513.9 \pm 279.0$ & & $744.3 \pm 514.2$ & $899.8 \pm 661.3$ \\
\hline \multicolumn{5}{|c|}{ CMJ (cm) } \\
\hline CWI (13) & $34.2 \pm 5.9$ & $29.3 \pm 6.0$ & $32.3 \pm 6.2$ & $33.4 \pm 6.5$ \\
\hline CWT (10) & $33.3 \pm 7.8$ & $29.2 \pm 7.0$ & $30.2 \pm 7.5$ & $31.4 \pm 8.0$ \\
\hline TWI (8) & $41.8 \pm 5.3$ & $35.5 \pm 4.3$ & $38.1 \pm 5.6$ & $40.1 \pm 3.9$ \\
\hline PAS (9) & $37.7 \pm 4.8$ & $32.0 \pm 6.4$ & $34.6 \pm 5.7$ & $36.9 \pm 6.5$ \\
\hline
\end{tabular}

Rupp et al. (2011)

\begin{tabular}{|c|c|c|c|c|}
\hline \multicolumn{5}{|c|}{ CMJ (cm) } \\
\hline & Baseline & Post-ex & $24 \mathrm{H}$ & $48 \mathrm{H}$ \\
\hline CWI (12) & $64.7+-11.7$ & $65.5+-10.0$ & $63.7+-9.8$ & $63.7+-8.8$ \\
\hline PAS (10) & $64.4+-10.70$ & $67.4+-12.3$ & $63.0+-10.4$ & $62.4+-10.4$ \\
\hline
\end{tabular}




\section{Annexe IV - Forest plots non pris en compte}

\section{DOMS à $24 H$}

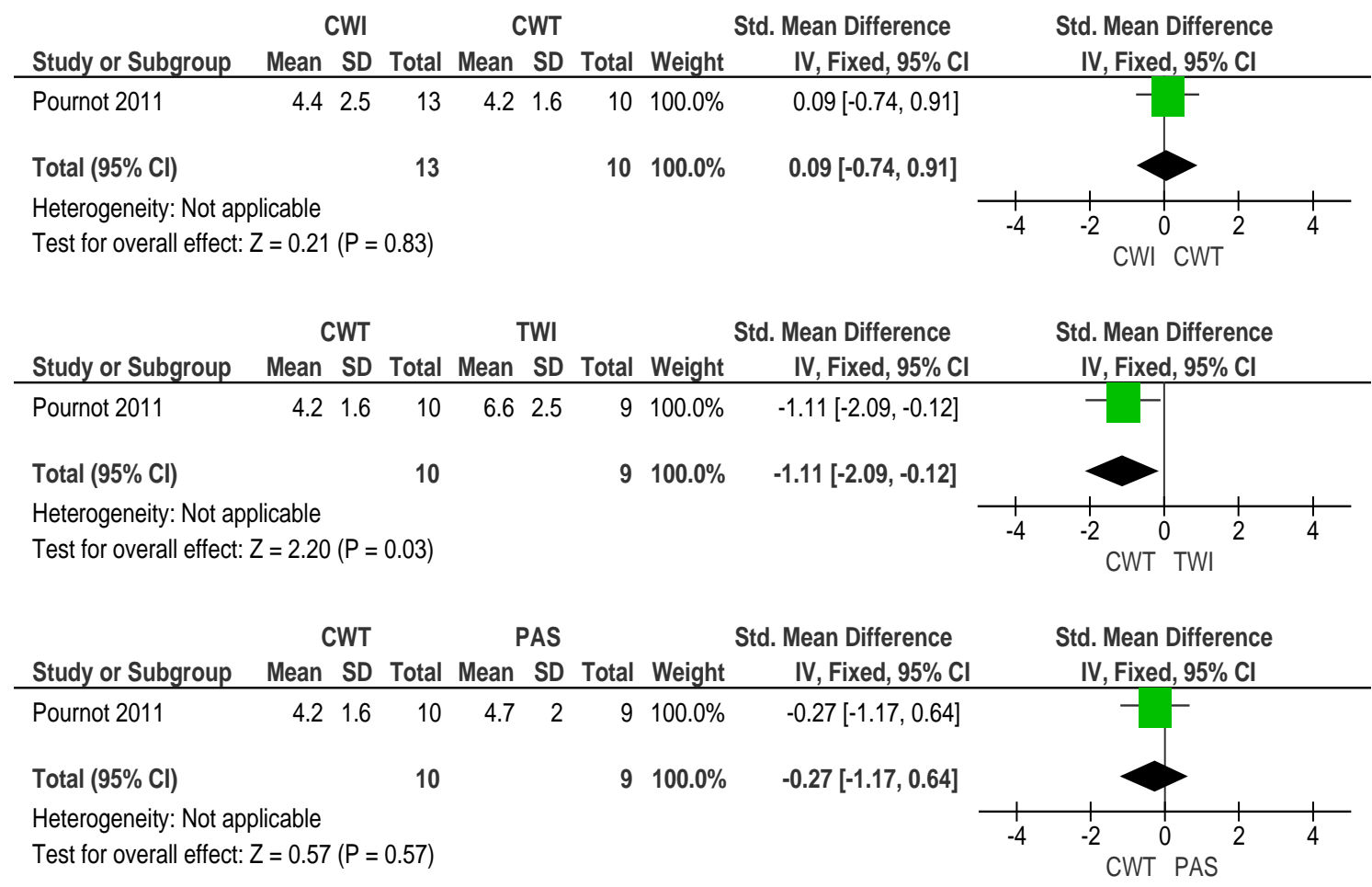

\section{CK à 24H}

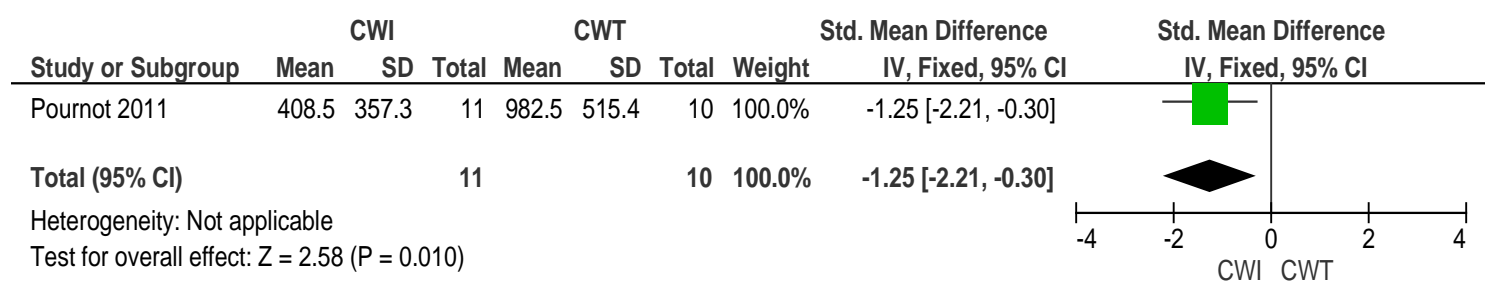

\begin{tabular}{|c|c|c|c|c|c|c|c|c|c|c|}
\hline \multirow[b]{2}{*}{ Study or Subgroup } & \multicolumn{3}{|c|}{ CWT } & \multicolumn{3}{|c|}{ TWI } & \multicolumn{2}{|c|}{ Std. Mean Difference } & \multirow{2}{*}{$\begin{array}{c}\text { Std. Mean Difference } \\
\text { IV, Fixed, } 95 \% \mathrm{Cl}\end{array}$} & \\
\hline & Mean & SD & Total & Mean & SD & Total & Weight & IV, Fixed, 95\% Cl & & \\
\hline Pournot 2011 & 982.5 & 515.4 & 10 & 834.6 & 492.3 & 9 & $100.0 \%$ & $0.28[-0.63,1.19]$ & & \\
\hline Total $(95 \% \mathrm{Cl})$ & & & 10 & & & 9 & $100.0 \%$ & $0.28[-0.63,1.19]$ & & \\
\hline $\begin{array}{l}\text { Heterogeneity: Not ar } \\
\text { Test for overall effect }\end{array}$ & $\begin{array}{l}\text { licable } \\
Z=0.61\end{array}$ & $(P=0$. & & & & & & & $\begin{array}{lc}-2 & 0 \\
& \text { CWT TWI }\end{array}$ & 4 \\
\hline
\end{tabular}

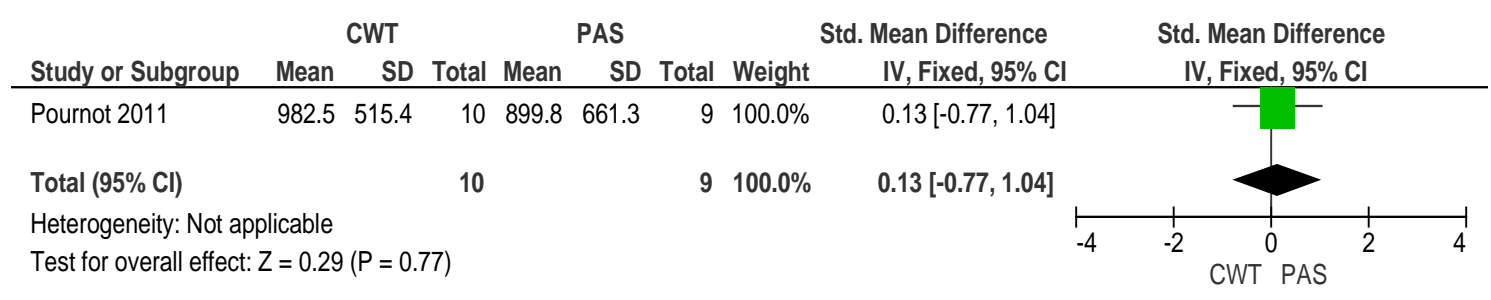




\section{Capacité fonctionnelle des membres inférieurs à $24 \mathrm{H}$}

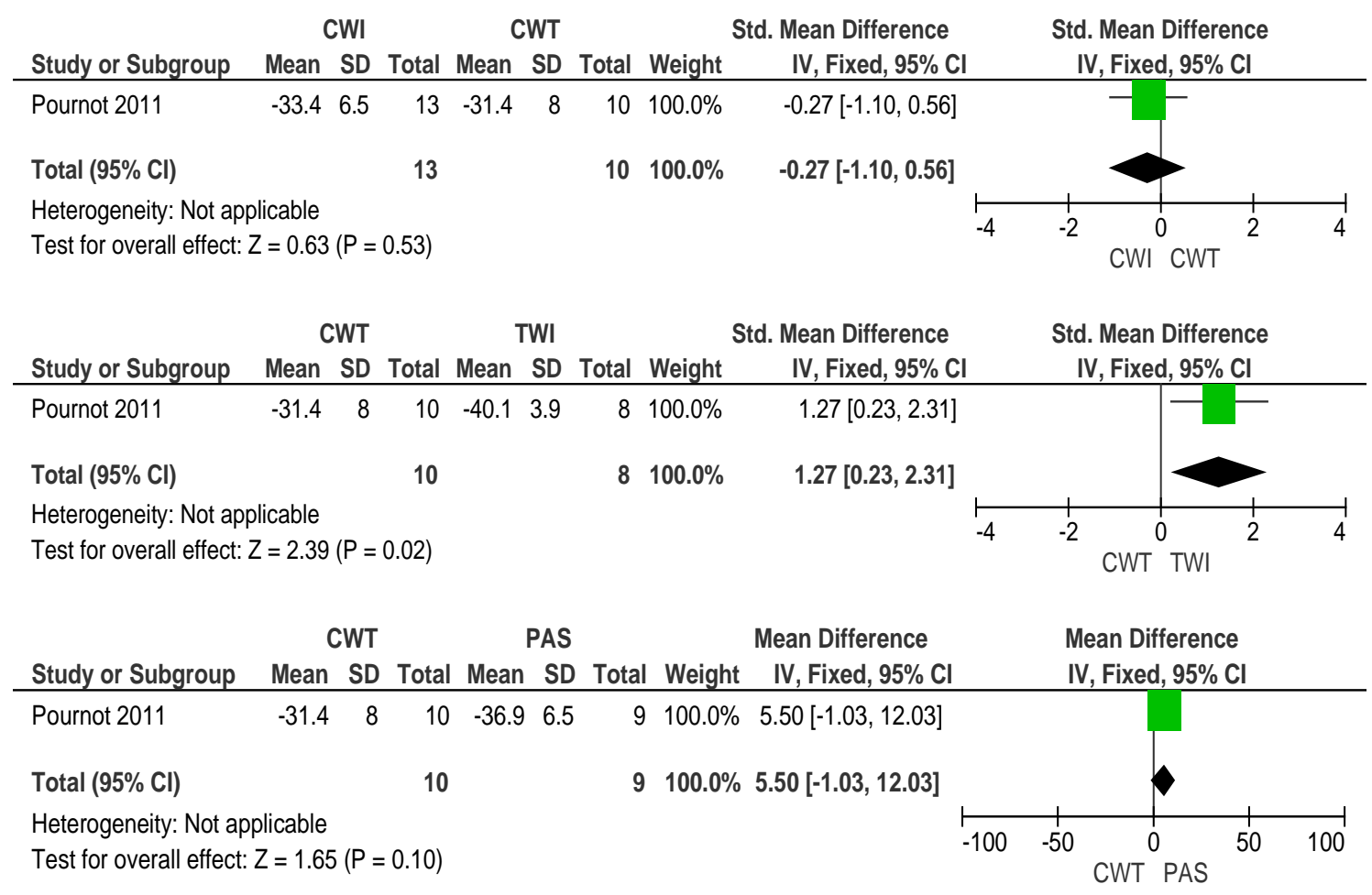




\title{
Les effets de l'immersion en eau froide ou contrastée sur la récupération post-effort dans les sports acycliques : une revue systématique et une méta-analyse
}

\author{
CARBONE P. ${ }^{a}$, HUMBERT-DROZ G. ${ }^{a}$, MATHIEU N.a,b \\ a Physiothérapeute, ${ }^{b}$ Chargé de cours à la HES-SO Valais
}

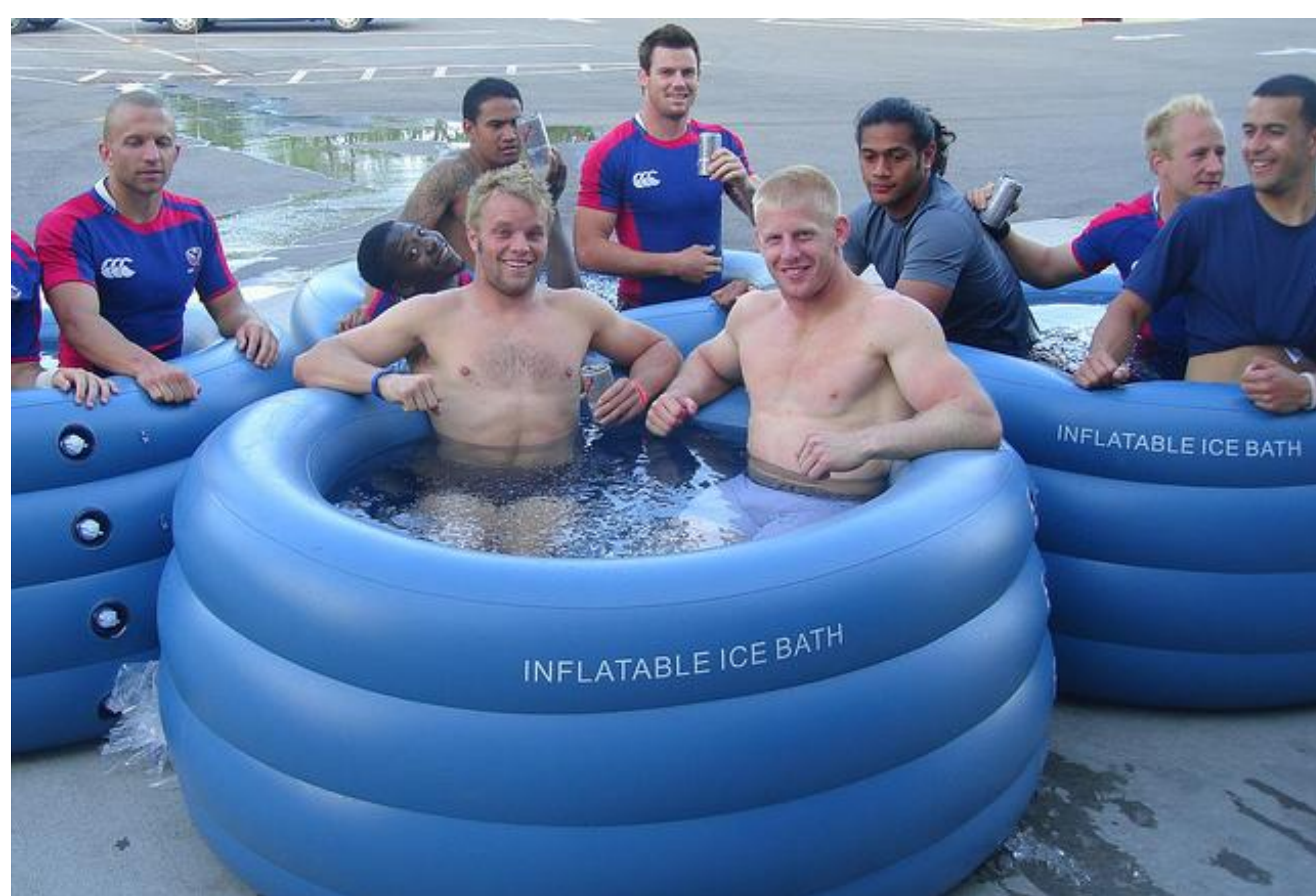

\section{Introduction}

Depuis quelques années, le monde du sport s'intéresse fortement à l'immersion comme technique de récupération suite à un exercice ${ }^{1}$. Malgré le manque de consensus scientifique, deux modalités d'immersion sont principalement utilisées: l'immersion en eau froide (Cold Water Immersion, CWI et en eau contrastée (Contrast Water Therapy, CWT).

\section{Objectif}

Évaluer les effets de ces moyens de récupération sur les courbatures (Delayed Onset Muscle Soreness, DOMS) le taux de créatine-kinase (CK) et la capacité fonctionnelle des membres inférieurs (hauteur de saut) dans des sports acycliques.

\section{Méthode}

Réalisation d'une revue systématique et d'une méta-analyse.

Consultation de quatre bases de données: 441 articles retrouvés.

\section{Critères d'inclusion:}

\section{- Sports acycliques}

- Immersion à hauteur des crêtes iliaques

- Présence d'au moins un des trois indicateurs

Au final, sélection de 4 études randomisées contrôlées ${ }^{2,3,4,5}$ suites aux étapes de tri et d'évaluation de la qualité méthodologique.

\section{Résultats}

Diminution statistiquement significative des DOMS à $24 \mathrm{H}$ pour la CWI.

Diminution statistiquement significative du taux de $\mathrm{CK}$ à $24 \mathrm{H}$ pour la $C W I$ par rapport à l'immersion en eau tempérée (TWI) et différence clinique en faveur de la $C W I$ par rapport à la récupération passive (PAS).

Différence statistiquement non significative pour la PAS par rapport à la $C W I$ mais aucune différence entre la $C W I$ et la $T W I$ pour la hauteur de saut à $24 \mathrm{H}$.

Utilisation d'une seule étude pour l'analyse narrative de la CWT ne montrant aucun résultat en faveur de cette modalité.

\section{Tableau récapitulatif des résultats pour la $C W I$}

\begin{tabular}{|c|c|c|}
\hline \multirow{2}{*}{ CK à 24H } & CWI vs PAS & CWI vs TWI \\
\hline \multirow{2}{*}{ Saut à 24H } & & \\
\cline { 2 - 3 } & & \\
\hline
\end{tabular}

1 En faveur de la $C W I$ (statistiquement significatif) $\quad$ En faveur de la PAS (statistiquement non-significatif) 1 En faveur de la CWI (statistiquement non-significatif) Aucune différence entre CWI et TWI

\section{Discussion}

Les résultats de notre étude sont en accord avec la plupart des publications.

Limites de notre revue:

- Échantillon total faible

- Une seule étude analysée pour la CWT

- Prise des indicateurs uniquement à $24 \mathrm{H}$

- Exercices pré-interventions variables

D'autres recherches sont nécessaires afin de déterminer un protocole d'immersion standardisé.

\section{Conclusion}

Notre travail démontre l'utilité de la $C W I$ dans la réduction des DOMS et du taux de CK post-effort dans des sports acycliques.

\section{Take Home Message}

* Utilisation recommandée de l'immersion en eau froide pour la récupération musculaire post-effort. * 10 à 15 minutes à une température comprise entre 10 et $12^{\circ} \mathrm{Celsius.}$

Contacts

Pierre Carbone, pierre.carbone@hotmail.com Gilles Humbert-Droz, gilles.humbert-droz@gmail.com

\section{Hes. SO IIV VaALIS}

